Worcester Polytechnic Institute

Digital WPI

Mathematical Sciences Faculty Publications

Department of Mathematical Sciences

10-1-2009

\title{
Approximating Infinity-Dimensional Stochastic Darcy's Equations without Uniform Ellipticity
}

J. Galvis

Marcus Sarkis

Worcester Polytechnic Institute, msarkis@wpi.edu

Follow this and additional works at: https://digitalcommons.wpi.edu/mathematicalsciences-pubs Part of the Mathematics Commons

\section{Suggested Citation}

Galvis, J., \& Sarkis, M. (2009). Approximating Infinity-Dimensional Stochastic Darcy's Equations without Uniform Ellipticity. SIAM Journal on Numerical Analysis, 47(5), 3624-3651. http://dx.doi.org/10.1137/080717924

This Article is brought to you for free and open access by the Department of Mathematical Sciences at Digital WPI. It has been accepted for inclusion in Mathematical Sciences Faculty Publications by an authorized administrator of Digital WPI. For more information, please contact digitalwpi@wpi.edu. 


\title{
APPROXIMATING INFINITY-DIMENSIONAL STOCHASTIC DARCY'S EQUATIONS WITHOUT UNIFORM ELLIPTICITY*
}

\author{
J. GALVIS ${ }^{\dagger}$ AND M. SARKIS $\ddagger$
}

\begin{abstract}
We consider a stochastic Darcy's pressure equation whose coefficient is generated by a white noise process on a Hilbert space employing the ordinary (rather than the Wick) product. A weak form of this equation involves different spaces for the solution and test functions and we establish a continuous inf-sup condition and well-posedness of the problem. We generalize the numerical approximations proposed in Benth and Theting [Stochastic Anal. Appl., 20 (2002), pp. 1191-1223] for Wick stochastic partial differential equations to the ordinary product stochastic pressure equation. We establish discrete inf-sup conditions and provide a priori error estimates for a wide class of norms. The proposed numerical approximation is based on Wiener-Chaos finite element methods and yields a positive definite symmetric linear system. We also improve and generalize the approximation results of Benth and Gjerde [Stochastics Stochastics Rep., 63 (1998), pp. 313-326] and Cao [Stochastics, 78 (2006), pp. 179-187] when a (generalized) process is truncated by a finite Wiener-Chaos expansion. Finally, we present numerical experiments to validate the results.
\end{abstract}

Key words. white noise analysis, Wiener-Chaos expansions, finite elements, stochastic partial differential equations, stochastic elliptic equations

AMS subject classifications. $60 \mathrm{H} 15,60 \mathrm{H} 30,60 \mathrm{H} 35,60 \mathrm{H} 40,65 \mathrm{M} 60,65 \mathrm{~N} 12,65 \mathrm{~N} 15,65 \mathrm{~N} 30$

DOI. $10.1137 / 080717924$

1. Introduction. A system of stochastic partial differential equations which models the two-phase flow in a porous medium arises in the mathematical modeling of transport of pollutants in groundwater and oil recovery processes. This system is composed of a transport equation for the saturation (the relative volume of one of the two fluids) coupled with an equation for the velocity field (given by Darcy's law and the incompressibility condition of the flow). The randomness enters the problem through the unknown properties of the rocks, especially the permeability tensor. In this paper we deal with one of the equations derived from this system; specifically, we consider an equation of the form

$$
\left\{\begin{aligned}
-\nabla_{x} \cdot\left(\kappa(x, \cdot) \nabla_{x} u(x, \cdot)\right) & =f(x, \cdot) \text { for all } x \in D \\
u(x, \cdot) & =0 \text { for all } x \in \partial D
\end{aligned}\right.
$$

where $\log \kappa(x, \cdot)$ is a Gaussian field and $f$ is a (possible random) forcing term; see Ghanem and Spanos [18], Babuška, Tempone, and Zouraris [3], Babuška, Nobile, and Tempone [2], Matthies and Keese [27], Furtado and Pereira [14], Roman and Sarkis [32], Frauenfelder, Schwab, and Todor [13], Xiu and Karniadakis [38], and the references therein. We emphasize that the assumption of the stochastic structure of the permeability function $\kappa(x, \cdot)$ is due to the lack of data and accuracy in the

${ }^{*}$ Received by the editors March 10, 2008; accepted for publication (in revised form) July 8, 2009; published electronically November 18, 2009.

http://www.siam.org/journals/sinum/47-5/71792.html

$\dagger$ Department of Mathematics, Texas A\&M University, College Station, TX 77843-3368 (jugal@ math.tamu.edu). This author's research was supported by PEC-PG-CAPES and CNPq Ph.D. fellowships.

¥Instituto Nacional de Matemática Pura e Aplicada (IMPA), Estrada Dona Castorina 110, CEP 22460-320, Rio de Janeiro, Brazil, and Department of Mathematical Sciences, Worcester Polytechnic Institute, 100 Institute Road, Worcester, MA 01609 (msarkis@wpi.edu). This author's research was supported in part by CNPQ Brazil under grant 300964/2006-4. 
measurements of the media. One approach that has been studied when the parameters in the equation are not completely known is the replacement of the true values of these parameters by some kind of average. By replacing the stochastic coefficient $\kappa(x, \cdot)$ in the equations by the average $\bar{\kappa}(x)$, we obtain some information about the solution, but usually this information is not enough to make more precise predictions of nonlinear functionals of the solution or to know what effect the small fluctuations in the parameter values actually have on the solution.

Another way to evaluate nonlinear functionals of the solution involves Monte Carlo approximations. Briefly speaking, Monte Carlo approximations require knowing the solution, or approximations of the solution, for many paths or realizations in the space of outcomes. In principle, if we know the distribution of the processes modeling the coefficients of the equation, we can simulate many trajectories of the coefficients, and for each trajectory we can apply a finite element method (FEM) to obtain an approximation of the solution for that particular realization. Then, Monte Carlo approximations of a nonlinear functional $g$ of the solution are of the form $\mathbb{E}[g(\hat{u}(x, \cdot))] \approx \frac{1}{M} \sum_{i=1}^{M} g\left(\hat{u}^{(a)}\left(x, \omega_{i}\right)\right)$, where $\mathbb{E}$ denotes the expectation operator, $M$ is the number of realizations, and $\hat{u}^{(a)}\left(x, \omega_{i}\right)$ is a finite element approximation of the solution at $x$ for the $i$ th-trajectory $\omega_{i}$. This procedure is, however, very time consuming as it involves assembling and solving large linear systems as many times as trajectories are simulated.

As alternatives to the Monte Carlo approach there are the methods that somehow "separate" the stochastic part from the deterministic part which have recently attracted the attention of several researchers; see $[1,2,3,8,13,16,17,18,22,23,26$, $27,29,28,33,37,38]$. To illustrate the advantage of these methods, let us suppose that the solution of (1.1)can be represented as

$$
\hat{u}(x, \omega)=\sum_{\alpha \in \mathcal{I}} \hat{u}_{\alpha}(x) Y_{\alpha}(\omega),
$$

where $\mathcal{I}$ is a countable index set and $\left\{Y_{\alpha}\right\}_{\alpha \in \mathcal{I}}$ is a collection of random variables with known probability distributions. Let us say we have an approximation of the solution of the form

$$
\hat{u}(x, \omega) \approx \hat{u}^{(a)}(x, \omega)=\sum_{\alpha \in \widetilde{\mathcal{I}}} \hat{u}_{\alpha}^{(a)}(x) Y_{\alpha}(\omega)
$$

where $\widetilde{\mathcal{I}}$ is a finite index set with $\widetilde{\mathcal{I}} \subset \mathcal{I}$. Assume that we have computed and stored the deterministic functions $\left\{\hat{u}_{\alpha}^{(a)}\right\}_{\alpha \in \tilde{\mathcal{I}}}$. Then, in order to approximate nonlinear functionals of the solution, we can either use the expression for $\hat{u}^{(a)}$ in (1.3) directly or use a Monte Carlo method based on (1.3). Note that when a simulation is required, all we need to do is to generate values for the random variables $\left\{Y_{\alpha}\right\}_{\alpha \in \tilde{\mathcal{I}}}$ and assemble the solution according to (1.3). In this way, we need to solve a very large linear system only once in order to compute the deterministic coefficients $\left\{\hat{u}_{\alpha}^{(a)}\right\}_{\alpha \in \tilde{\mathcal{I}}}$. The effectiveness of this procedure depends mainly on the following:

1. The kind of expansion used in (1.2) and (1.3). Usually a Wiener-Chaos expansion is considered.

2. The finite dimensional problem involved in the computation of the coefficients $\left\{\hat{u}_{\alpha}^{(a)}\right\}_{\alpha \in \tilde{\mathcal{J}}}$ in (1.3). Usually a Galerkin or Petrov-Galerkin type problem that uses the original coefficient $\kappa$ or an approximation of it, for instance, a truncated Karhúnen-Loève or a Wiener-Chaos expansion, is considered. 
The Karhúnen-Loève (KL) expansion of a stochastic process with continuous covariance function is well-used in many engineering applications as an efficient tool to characterize and store the random process associated with the permeability or forcing terms. Its usage comes from the fact that the truncated KL expansion is optimal in the $L^{2}$ sense since it minimizes the mean square error. An issue inherited in the KL expansion is the possible excess of oscillations of the terms of the expansion near the boundary $\partial D$. The KL expansion also requires the computation of the eigenvalues and eigenfunctions of the covariance operator associated with $\kappa$ or $\log \kappa$; see $[13,33]$ and the references therein.

The approximation of solutions of partial differential equations based on WienerChaos expansion and white noise analysis has been considered in the literature; see [11, $20,21,25]$ and the references therein. The Wiener-Chaos expansion is the orthogonal expansion, in terms of Fourier-Hermite stochastic polynomials, of random processes defined in the white noise space. The underlying white noise space can be constructed based on convolution kernels on $L^{2}\left(\mathbb{R}^{d}\right)$ or on KL expansions on $L^{2}(D)$. Using these constructions, analytical and algebraic properties can be explored to define norms and to improve the complexity of the algorithms; see section 7 for a unified description of how to construct white noise spaces. The corresponding white noise analysis is fundamental for establishing stochastic regularity results and approximation results. We now refer to some of the works that have inspired this paper.

We mention Benth and Theting [5] and Theting [35], where they analyze the stochastic pressure equation when the ordinary product is replaced by the Wick product. These papers also consider other Wick stochastic partial differential equations. They use the white noise calculus and propose an approximation by truncating the Wiener-Chaos expansion of the solution. They present a priori error estimates based on the work of Benth and Gjerde [4] on estimating errors when truncating a WienerChaos expansion. We also mention Cao [9], where the estimates in [4] are improved. Roman and Sarkis [32] present and explain several features and advantages of using white noise calculus as a natural framework for the study of the stochastic pressure equation without replacing the ordinary product by the Wick product. They consider a permeability process $\kappa(x, \omega ; \phi)=\rho_{0}+e^{W_{\phi}(x, \omega)}$, where $W_{\phi}(x, \omega)$ is the 1 dimensional smoothed white noise process defined on the 1-dimensional white noise probability space $\left(\mathcal{S}^{\prime}\left(\mathbb{R}^{d}\right), \mathcal{B}\left(\mathcal{S}^{\prime}\left(\mathbb{R}^{d}\right)\right), \mu\right)$; see section 7.1.1. The constant $\rho_{0}>0$ is added to guarantee uniform ellipticity and a priori error estimates are not provided.

In this paper, we consider $\rho_{0}=0$ and the ordinary product in infinity dimensions. We provide a priori error estimates for a class of smoothed white noise processes $W_{\phi}(x, \omega)$. We consider the white noise calculus constructed from a Hilbert space and an operator in order to define adequate spaces for proving the existence and uniqueness of the solution of the ordinary product stochastic pressure equation. When formulating the weak version of the problem (1.1) we choose different spaces for the solution and test functions in order to circumvent the uniform ellipticity. The norms to define these spaces take into account the stochastic exponential decay (or growth) of the functions and depend on the white noise probability space (which depends on $\kappa(x, \omega))$ and on the stochastic exponential behavior of the forcing term $f(x, \omega)$. The well-posedness of the problem is then established via inf-sup techniques. For the finite dimensional problem we also use different spaces for the solution and the test functions, however, yielding a positive definite symmetric linear system. The wellposedness of the discrete problem is also established via discrete inf-sup techniques. To derive the discrete inf-sup condition and a priori error estimates, we consider norms of Hida-Kuo-Kondratiev-Streit type which depend on the choice of sequence 
of weights; see Hida [19], Kuo [25], Holden et al. [21], Hida et al. [20], Da Prato [11], and the references therein. For some particular choices, these norms measure the regularity of the process in the white noise probability space just as the Sobolev norms measure regularity of functions on spatial variable. We also point out that we adopt a general setup which permits a unified analysis of several modeling choices of smoothed white noise processes. For regularity results of the pressure equation for these type of norms, we refer to Theting [35] and [15].

We mention that a different approach to ours is considered in Babuška, Nobile, and Tempone [2]. By using $L_{p}$ spaces for the probability space, they show the wellposedness for problems where the permeability coefficient is, for instance, a finite dimensional lognormal. They consider a coefficient of the form of $\kappa(x, \omega)=\rho_{0}+$ $e^{\sum_{j=1}^{K} a_{j}(x) Y_{j}(\omega)}$, where $\rho_{0}$ is a positive constant, and assume that each deterministic function $a_{j}$ is bounded in $D$. Their theoretical results depend on $\rho_{0}$ and on the value of $\sum_{j=1}^{K} \sup _{x \in D} a_{j}(x)^{2}$. Using our approach, we can consider the case $\rho_{0}=0$ (without uniform ellipticity) and the infinity number of KL terms and the assumption required to show well-posedness is $\sup _{x \in D} \sum_{j=1}^{\infty} \lambda_{j}^{2 \theta} a_{j}(x)^{2}<\infty$. Here $\theta>0$ and $\left\{\lambda_{j}\right\}_{j=1}^{\infty}$ is any sequence with $1<\lambda_{1} \leq \lambda_{2} \leq \cdots$ and $\sum_{j=1}^{\infty} \lambda_{j}^{-2 \theta}<\infty$; see Theorem 3.1.

This paper is structured as follows. In section 2 we introduce the white noise calculus framework to be used in the rest of the paper. In section 3 we introduce the adequate spaces for the solution and test functions of the stochastic pressure equation and establish its well-posedness. These spaces are characterized in section 4 where additional norms are introduced in order to measure the regularity in the $\omega$ variable. Two examples of such norms are presented. In section 5 we consider a Galerkin approximation and deduce a priori error estimates. The resulting linear system is studied in section 6 . Section 7 discusses some modeling choices, and finally, in section 8 we present a 1-dimensional numerical experiment.

2. Framework: White noise analysis. Let $H$ be a real separable Hilbert space with inner product $(\cdot, \cdot)_{H}$ and norm $\|\cdot\|_{H}$. Let $A$ be an operator on $H$ such that there exists an $H$-orthonormal basis $\left\{\eta_{j}\right\}_{j=1}^{\infty}$ satisfying the following:

1) $A \eta_{j}=\lambda_{j} \eta_{j}, j=1,2, \ldots$

2) $1<\lambda_{1} \leq \lambda_{2} \leq \cdots$.

3) $\sum_{j=1}^{\infty} \lambda_{j}^{-2 \theta}<\infty$ for some constant $\theta>0$.

For $p>0$ let $\mathcal{S}_{p}:=\left\{\xi \in H ;\|\xi\|_{p}<\infty\right\}$, where

$$
\|\xi\|_{p}^{2}:=\left\|A^{p} \xi\right\|_{H}^{2}=\sum_{j=1}^{\infty} \lambda_{j}^{2 p}\left(\xi, \eta_{j}\right)_{H}^{2}
$$

and for $p<0$ let $\mathcal{S}_{p}$ be defined as the dual space of $\mathcal{S}_{-p}$. It is easy to see that for $p<0$ we have $\|\cdot\|_{p}=\left\|A^{p} \cdot\right\|_{H}$, i.e., $\|\xi\|_{p}^{2}=\sum_{j=0}^{\infty} \lambda_{j}^{2 p}\left\langle\xi, \eta_{j}\right\rangle^{2}$ for all $p<0$ and $\xi \in \mathcal{S}_{p}$, and the duality pairing between $\mathcal{S}_{p}$ and $\mathcal{S}_{-p}$ is an extension of the $H$ inner product. We also define

$$
\mathcal{S}:=\cap_{p \geq 0} \mathcal{S}_{p} \text { (with the projective limit topology), }
$$

and let $\mathcal{S}^{\prime}$ be defined as the dual space of $\mathcal{S}$, i.e., by considering the standard countably Hilbert space constructed from $(H, A)$; see Kuo [25] and Obata [30].

Let $\left(\mathcal{S}^{\prime}, \mathcal{B}\left(\mathcal{S}^{\prime}\right), \mu\right)$ be the probability space with the sigma-field $\mathcal{B}\left(\mathcal{S}^{\prime}\right)$ of Borel subsets of $\mathcal{S}^{\prime}$. The probability measure $\mu$ is given by the Bochner-Minlos theorem 
and characterized by

$$
E_{\mu} e^{i\langle\cdot, \xi\rangle}:=\int_{\mathcal{S}^{\prime}} e^{i\langle\omega, \xi\rangle} d \mu(\omega)=e^{-\frac{1}{2}\|\xi\|_{H}^{2}} \text { for all } \xi \in \mathcal{S} .
$$

Here, the pairing $\langle\omega, \xi\rangle=\omega(\xi)$ is the action of $\omega \in \mathcal{S}^{\prime}$ on $\xi \in \mathcal{S}$, and $E_{\mu}$ denotes the expectation with respect to the measure $\mu$; see Obata [30, Chapters 1-3], Holden et al. [21, Chapter 2], Hida [19, Chapter 3], Hida et al. [20], Kuo [25], and Berezanskiu [6]. The measure $\mu$ is often called the (normalized) Gaussian measure on $\mathcal{S}^{\prime}$ due to the following remark.

Remark 2.1. Equation (2.1) says that for any test function $\xi \in \mathcal{S}$, the random variable $\langle\cdot, \xi\rangle$ is normally distributed with zero mean and variance $\|\xi\|_{H}^{2}$. If $\xi_{1}, \ldots, \xi_{j} \in$ $\mathcal{S}$ are orthonormal in $H$, then the random variables $\left\langle\cdot, \xi_{1}\right\rangle, \ldots,\left\langle\cdot, \xi_{j}\right\rangle$ are independent and normally distributed with mean zero and variance equal to one; see Holden et al. [21], Kuo [25], and Obata [30].

The following particular case of Fernique's theorem will be used throughout this paper; see Shigekawa [34], Bogachev [7], Kuo [24], Da Prato [11], and Da Prato and Zabczyk [12].

LEMMA 2.2. We have

$$
\int_{\mathcal{S}^{\prime}} e^{s\|\omega\|_{-\theta}^{2}} d \mu(\omega)= \begin{cases}\prod_{j=1}^{\infty}\left(1-\frac{2 s}{\lambda_{j}^{2 \theta}}\right)^{-\frac{1}{2}}, & s<\frac{\lambda_{1}^{2 \theta}}{2}, \\ +\infty, & s \geq \frac{\lambda_{1}^{2 \theta}}{2} .\end{cases}
$$

Proof. Let $s<\frac{\lambda_{1}^{2 \theta}}{2}$. Note that $\|\omega\|_{-\theta}^{2}=\sum_{j=1}^{\infty} \lambda_{j}^{-2 \theta}\left\langle\omega, \eta_{j}\right\rangle^{2}$. Using the monotone convergence theorem when $s>0$ or the dominated convergence theorem when $s<0$, we have

$$
\begin{aligned}
\int_{\mathcal{S}^{\prime}} e^{s\|\omega\|_{-\theta}^{2}} d \mu(\omega)=\lim _{J \rightarrow \infty} \int_{\mathcal{S}^{\prime}} e^{s \sum_{j=1}^{J} \lambda_{j}^{-2 \theta}\left\langle\omega, \eta_{j}\right\rangle^{2}} d \mu(\omega) \\
=\lim _{J \rightarrow \infty} \prod_{j=1}^{J} \frac{1}{\sqrt{2 \pi}} \int_{\mathbb{R}} e^{s \lambda_{j}^{-2 \theta} y^{2}} e^{-\frac{1}{2} y^{2}} d y \\
=\lim _{J \rightarrow \infty} \prod_{j=1}^{J} \frac{1}{\sqrt{2 \pi}} \int_{\mathbb{R}} \exp \left(-\frac{1}{2}\left(1-\frac{2 s}{\lambda_{j}^{2 \theta}}\right) y^{2}\right) d y=\prod_{j=1}^{\infty}\left(1-\frac{2 s}{\lambda_{j}^{2 \theta}}\right)^{-\frac{1}{2}} .
\end{aligned}
$$

In view of the assumption $\sum_{j=1}^{\infty} \lambda_{j}^{-2 \theta}<\infty$, the infinite product above converges when $s<\frac{\lambda_{1}^{2 \theta}}{2}$ and goes to $+\infty$ when $s$ approaches $\frac{\lambda_{1}^{2 \theta}}{2}$ from below. Because the integral above is monotonically increasing with respect to $s$, the lemma follows.

We note that if $\sum_{j=1}^{\infty} \lambda_{j}^{-2 \theta}<\infty$, then Lemma 2.2 implies $\int_{\mathcal{S}^{\prime}}\|\omega\|_{-\theta}^{2} d \mu(\omega)<\infty$, which in turn implies that $\mu\left(\mathcal{S}_{-\theta}\right)=1$. To see this, note that $\mathcal{S}^{\prime} \backslash \mathcal{S}_{-\theta}=\left\{\omega:\|\omega\|_{-\theta}^{2}=\right.$ $\infty\}$, and then $\mu\left(\mathcal{S}^{\prime} \backslash \mathcal{S}_{-\theta}\right)>0$ would imply that $\int_{S^{\prime}}\|\omega\|_{-\theta}^{2} d \mu(\omega)=\infty$, which gives a contradiction. Without further comments, we use that $\mu\left(\mathcal{S}_{-\theta}\right)=1$ throughout this paper.

We now define the generalized stochastic processes $w(\xi, \omega) \in \mathbb{R}, \xi \in \mathcal{S}, \omega \in \mathcal{S}^{\prime}$.

Definition 2.3 (see Holden et al. [21]). The 1-dimensional or scalar white noise associated with $H$ and $A$ is the map $w: \mathcal{S} \times \mathcal{S}^{\prime} \longrightarrow \mathbb{R}$ given by $w(\xi, \omega)=\langle\omega, \xi\rangle$ for $\omega \in \mathcal{S}^{\prime}, \xi \in \mathcal{S}$.

It is not difficult to prove that when $\xi \in H$ and we choose any $\xi_{n} \in \mathcal{S}$ such that $\xi_{n} \rightarrow \xi$ in $H$, then $\langle\omega, \xi\rangle:=\lim _{n \rightarrow+\infty}\left\langle\omega, \xi_{n}\right\rangle$ exists in $L^{2}(\mu)$, and is independent of the 
choice of $\left\{\xi_{n}\right\}_{n=1}^{\infty}$. Thus, the definition of white noise can be extended to functions in $H$. In what follows we use the notation $\left(L^{2}\right)$ or $\left(L^{2}\right)_{0}$ for the space $L^{2}(\mu)$. We always interpret properties in the "almost everywhere" or "almost surely" or "almost all" sense; therefore, we will sometimes omit this interpretation to make notation and formula less cumbersome.

We restrict our considerations to the 1-dimensional smoothed white noise case.

Definition 2.4. Let $D \subset \mathbb{R}^{d}$. Using the map $w$ of Definition 2.3 we can construct a stochastic process, called the smoothed white noise process $W_{\phi}(x, \omega)$, as follows:

$$
W_{\phi}(x, \omega):=w\left(\phi_{x}, \omega\right)=\left\langle\omega, \phi_{x}\right\rangle, x \in D, \omega \in \mathcal{S}^{\prime},
$$

where $\phi_{x} \in H$ for all $x \in D$. Examples of $\phi_{x}$ are given in Examples 2.6 and 2.7 below, and in more detail in section 7 . We point out that the smoothed white noise formalism unifies the analysis of stochastic processes obtained via KL expansions (see section 7.2.3) or via convolution methods (see section 7.2.1).

Remark 2.5. The terminology smoothed white noise comes from the fact that the process $\left\{W_{\phi}(x, \cdot)\right\}_{x \in D}$ has the following properties:

(i) For each $x \in D, W_{\phi}(x, \cdot)$ is normally distributed with zero mean and variance $\left\|\phi_{x}\right\|_{H}^{2}$.

(ii) For each $x$ and $\hat{x} \in D$ we have $E_{\mu} W_{\phi}(x, \cdot) W_{\phi}(\hat{x}, \cdot)=\left(\phi_{x}, \phi_{\hat{x}}\right)_{H}$.

Example 2.6. We can take $H=L^{2}(\mathbb{R})$ and $A=-\frac{d^{2}}{d x^{2}}+x^{2}+1$. In this case the eigenfunction $\eta_{j}$ is the $j$ th Hermite function and $\lambda_{j}=2 j$; see section 7.1 and (7.2). Let $D=[0,1]$, and let $\phi_{x}(\hat{x})=\phi(\hat{x}-x), x \in D$, and $\hat{x} \in \mathbb{R}$, where the window $\phi$ can be chosen such that the diameter of supp $\phi$ is the maximum distance with which $W_{\phi}(x, \cdot)$ and $W_{\phi}(\hat{x}, \cdot)$ might be correlated; see Holden et al. [21].

Example 2.7. Let $D \subset \mathbb{R}^{d}$ and take $H=L^{2}(D)$ and $A=Q^{-1}$, where $Q$ : $L^{2}(D) \rightarrow L^{2}(D)$ is the integral operator on $(D \times D)$ with kernel given by a covariance $C(x, \hat{x})$. In this case, for $x, \hat{x} \in D$, we define $\phi_{x}(\hat{x})=\sum_{j=1}^{\infty} \lambda_{j}{ }^{-1 / 2} \eta_{j}(x) \eta_{j}(\hat{x})$, where $\lambda_{j}$ and $\eta_{j}$ are the eigenvalues and eigenfunctions of $A$. It is easy to see that $E_{\mu}\left(W_{\phi}(x, \cdot) W_{\phi}(\hat{x}, \cdot)\right)=C(x, \hat{x})$; see section 7.2.3.

3. The problem and variational formulation. Given $\phi: D \rightarrow \mathcal{S}_{\theta}$ we consider the following problem: For all $\omega \in \mathcal{S}^{\prime}$, find $u(x, \omega)$ such that

$$
\left\{\begin{aligned}
-\nabla_{x} \cdot\left(\kappa(x, \omega) \nabla_{x} u(x, \omega)\right) & =f(x, \omega) \text { for all } x \in D \\
u(x, \omega) & =0 \text { for all } x \in \partial D
\end{aligned}\right.
$$

where

$$
\kappa(x, \omega):=e^{W_{\phi}(x, \omega)}=e^{\left\langle\omega, \phi_{x}\right\rangle}, \quad x \in D,
$$

and the exponent $W_{\phi}(x, \omega)$ is the 1-dimensional smoothed white noise process of Definition 2.4. Thus, $\kappa$ is a lognormal random process. Observe that for different families $\left\{\phi_{x} \in \mathcal{S}_{\theta}\right\}_{x \in D}$ there exists a different permeability function $\kappa(\cdot, \cdot)$ associated with it, and therefore, the solution $u(\cdot, \cdot)$ depends on $\phi$.

To motivate the definition of the spaces for the solution of (3.1), observe that since $\mu\left(\mathcal{S}_{-\theta}\right)=1$ and $\phi_{x} \in \mathcal{S}_{\theta}$ for all $x \in D$, we can write

$$
\left|\left\langle\omega, \phi_{x}\right\rangle\right| \leq\|\omega\|_{-\theta} \sup _{x \in D}\left\|\phi_{x}\right\|_{\theta}, \quad \omega \text {-a.s. in } \mathcal{S}^{\prime} .
$$


Denote $C_{\theta}=C_{\theta}(\phi):=\sup _{x \in D}\left\|\phi_{x}\right\|_{\theta}$. Then we have for all $\epsilon>0$

$$
-\frac{\epsilon}{2}\|\omega\|_{-\theta}^{2}-\frac{C_{\theta}^{2}}{2 \epsilon} \leq-\|\omega\|_{-\theta}^{2} C_{\theta} \leq\left\langle\omega, \phi_{x}\right\rangle \leq\|\omega\|_{-\theta}^{2} C_{\theta} \leq \frac{\epsilon}{2}\|\omega\|_{-\theta}^{2}+\frac{C_{\theta}^{2}}{2 \epsilon}
$$

and

$$
\kappa_{\min }(\omega):=e^{-\frac{C_{\theta}^{2}}{2 \epsilon}} e^{-\frac{\epsilon}{2}|\omega|_{-\theta}^{2}} \leq \kappa(x, \omega) \leq e^{\frac{C_{\theta}^{2}}{2 \epsilon}} e^{\frac{\epsilon}{2}\|\omega\|_{-\theta}^{2}}=: \kappa_{\max }(\omega) .
$$

We now motivate the definitions of the solution and test function spaces. When $u(\cdot, \omega)$ is the weak solution of (3.1) for almost all $\omega \in \mathcal{S}^{\prime}$, then from the Lax-Milgram lemma we have

$$
|u(\cdot, \omega)|_{H_{0}^{1}(D)}^{2} \leq \frac{1}{\kappa_{\min }(\omega)^{2}}\|f(\cdot, \omega)\|_{H^{-1}(D)}^{2}=e^{\frac{C_{\theta}^{2}}{\epsilon}} e^{\epsilon\|\omega\|_{-\theta}^{2}}\|f(\cdot, \omega)\|_{H^{-1}(D)}^{2} .
$$

Then for $s \in \mathbb{R}$ we can write

$$
|u(\cdot, \omega)|_{H_{0}^{1}(D)}^{2} e^{s\|\omega\|_{-\theta}^{2}} \leq e^{\frac{C_{\theta}^{2}}{\epsilon}}\|f(\cdot, \omega)\|_{H^{-1}(D)}^{2} e^{(s+\epsilon)|\omega|_{-\theta}^{2}} \quad \text { a.s. in } \mathcal{S}^{\prime},
$$

and integrating both sides we obtain

$$
\int_{\mathcal{S}^{\prime}}|u(\cdot, \omega)|_{H_{0}^{1}(D)}^{2} e^{s\|\omega\|_{-\theta}^{2}} d \mu(\omega) \leq e^{\frac{C_{\theta}^{2}}{\epsilon}} \int_{\mathcal{S}^{\prime}}\|f(\cdot, \omega)\|_{H^{-1}(D)}^{2} e^{(s+\epsilon)\|\omega\|_{-\theta}^{2}} d \mu(\omega) .
$$

This last inequality gives us an idea of the spaces where we can seek the solution and choose the test functions. For the solution space we use the left-hand-side norm given in (3.4), while for the test function spaces we use the right-hand-side dual norm in (3.4).

Define $\mathcal{U}_{s}^{m}$ as the space of functions $u: D \times \mathcal{S}^{\prime} \rightarrow \mathbb{R}$ such that

$$
\int_{\mathcal{S}^{\prime}}\|u(\cdot, \omega)\|_{H^{m}(D)}^{2} e^{s\|\omega\|_{-\theta}^{2}} d \mu(\omega)<+\infty
$$

with norm $\|u\|_{\mathcal{U}_{s}^{m}}^{2}:=\int_{\mathcal{S}^{\prime}}\|u(\cdot, \omega)\|_{H^{m}(D)}^{2} e^{s\|\omega\|_{-\theta}^{2}} d \mu(\omega)$ and corresponding seminorm $|u|_{\mathcal{U}_{s}^{m}}^{2}:=\int_{\mathcal{S}^{\prime}}|u(\cdot, \omega)|_{H^{m}(D)}^{2} e^{s\|\omega\|_{-\theta}^{2}} d \mu(\omega)$.

Note that $\mathcal{U}_{0}^{0}=L^{2}(D) \otimes\left(L^{2}\right)_{0}$, where $\left(L^{2}\right)_{0}=L^{2}\left(\mathcal{S}^{\prime}, \mu(\omega)\right)$, and in general, we have $\mathcal{U}_{s}^{m}=H^{m}(D) \otimes\left(L^{2}\right)_{s}$, where

$$
\left(L^{2}\right)_{s}:=L^{2}\left(\mathcal{S}^{\prime}, e^{s\|\omega\|_{-\theta}^{2}} d \mu(\omega)\right)
$$

with norm $\|v\|_{\left(L^{2}\right)_{s}}^{2}:=\int_{\mathcal{S}^{\prime}}|v(\omega)|^{2} e^{s\|\omega\|_{-\theta}^{2}} d \mu(\omega)$. We also define $\widehat{\mathcal{U}}_{s}^{1}:=H_{0}^{1}(D) \otimes\left(L^{2}\right)_{s} \subset$ $\mathcal{U}_{s}^{1}$, i.e., the functions in $\mathcal{U}_{s}^{1}$ which vanish on $\partial D$ a.s. in $\omega$. By using a Poincaré inequality, the seminorm $|\cdot|_{\mathcal{U}_{s}^{1}}$ is a norm equivalent to $\|\cdot\|_{\mathcal{U}_{s}^{1}}$ in $\widehat{\mathcal{U}}_{s}^{1}$. Since the space $\left(L^{2}\right)_{s+\epsilon}$ is the dual of $\left(L^{2}\right)_{-s-\epsilon}$ and the $H^{-1}(D)$ is the dual of $H_{0}^{1}(D)$, we can identify the dual space of $\widehat{\mathcal{U}}_{-s-\epsilon}^{1}$ with $\mathcal{U}_{s+\epsilon}^{-1}$, where the duality pairing is given by

$$
\langle f, v\rangle:=\int_{\mathcal{S}^{\prime}}\langle f(\cdot, \omega), v(\cdot, \omega)\rangle_{H^{-1}, H^{1}} d \mu(\omega) \quad \text { for all } v \in \widehat{\mathcal{U}}_{-s-\epsilon}^{1}, \quad f \in \mathcal{U}_{s+\epsilon}^{-1} .
$$

Here $\langle\cdot, \cdot\rangle_{H^{-1}, H^{1}}$ denotes the duality pairing between $H^{-1}(D)$ and $H_{0}^{1}(D)$. 
We define the bilinear form $a: \widehat{\mathcal{U}}_{s}^{1} \times \widehat{\mathcal{U}}_{-s+\epsilon}^{1} \rightarrow \mathbb{R}$ by

$$
a(u, v):=\int_{D \times \mathcal{S}^{\prime}} \kappa(x, \omega) \nabla u(x, \omega) \nabla v(x, \omega) d x d \mu(\omega),
$$

and let $T_{a}: \widehat{\mathcal{U}}_{s}^{1} \rightarrow \mathcal{U}_{s-\epsilon}^{-1}$ be the linear operator defined by

$$
a(u, v)=\left\langle T_{a} u, v\right\rangle \quad \text { for all } u \in \widehat{\mathcal{U}}_{s}^{1}, \quad v \in \widehat{\mathcal{U}}_{-s+\epsilon}^{1} .
$$

We will show in the next theorem that $T_{a}$ is a continuous operator. We also define the domain $\widehat{\mathcal{D}}_{s}^{1} \subset \widehat{\mathcal{U}}_{s}^{1}$ by

$$
\widehat{\mathcal{D}}_{s}^{1}:=\left\{u \in \widehat{\mathcal{U}}_{s}^{1}: \sup _{v \in \widehat{\mathcal{U}}_{-s-\epsilon}^{1} \backslash\{0\}} \frac{a(u, v)}{|v|_{\mathcal{U}_{-s-\epsilon}^{1}}}<\infty\right\} ;
$$

i.e., the $u \in \widehat{\mathcal{U}}_{s}^{1}$ such that $T_{a} u \in \mathcal{U}_{s+\epsilon}^{-1}$. We note that $\widehat{\mathcal{D}}_{s}^{1}$ is not empty since we will show in the next theorem that $\widehat{\mathcal{U}}_{s+2 \epsilon}^{1} \subset \widehat{\mathcal{D}}_{s}^{1}$.

The weak formulation of problem (3.1) is introduced as follows:

$$
\left\{\text { Find } \hat{u} \in \widehat{\mathcal{D}}_{s}^{1} \subset \widehat{\mathcal{U}}_{s}^{1} \text { such that } a(\hat{u}, v)=\langle f, v\rangle \text { for all } v \in \widehat{\mathcal{U}}_{-s-\epsilon}^{1},\right.
$$

where the duality pairing between $f \in \mathcal{U}_{s+\epsilon}^{-1}$ and $v \in \widehat{\mathcal{U}}_{-s-\epsilon}$ is given by

$$
\langle f, v\rangle=\int_{D \times \mathcal{S}^{\prime}}\langle f(\cdot, \omega), v(\cdot, \omega)\rangle_{H^{-1}, H^{1}} d \mu(\omega) .
$$

Theorem 3.1 (well-posedness). Let $\epsilon>0$ and $\phi: D \rightarrow \mathcal{S}_{\theta}$ with $C_{\theta}:=$ $\sup _{x \in D}\left\|\phi_{x}\right\|_{\theta}<\infty$. Then we have the following results:

(1) The bilinear form $a: \widehat{\mathcal{U}}_{s}^{1} \times \widehat{\mathcal{U}}_{-s+\epsilon}^{1} \rightarrow \mathbb{R}$ is continuous and we have

$$
a(u, v) \leq e^{\frac{C_{\theta}^{2}}{2 \epsilon}}|u|_{\mathcal{U}_{s}^{1}}|v|_{\mathcal{U}_{-s+\epsilon}^{1}} \quad \text { for all } u \in \widehat{\mathcal{U}}_{s}^{1}, v \in \widehat{\mathcal{U}}_{-s+\epsilon}^{1} .
$$

(2) The bilinear form a satisfies the following inf-sup condition:

$$
\inf _{u \in \widehat{\mathcal{U}}_{s}^{1} \backslash\{0\}} \sup _{v \in \widehat{\mathcal{U}}_{-s-\epsilon}^{1} \backslash\{0\}} \frac{a(u, v)}{|u|_{\mathcal{U}_{s}^{1}}^{1}|v|_{\mathcal{U}_{-s-\epsilon}^{1}}^{1}} \geq e^{-\frac{C_{\theta}^{2}}{2 \epsilon}} .
$$

(3) For any $v \in \widehat{\mathcal{U}}_{-s-\epsilon}^{1} \backslash\{0\}$ there exists $u(v) \in \widehat{\mathcal{U}}_{s+2 \epsilon}^{1}$ such that $a(u, v) \neq 0$.

(4) For any $f \in \mathcal{U}_{s+\epsilon}^{-1} \subset \mathcal{U}_{s-\epsilon}^{-1}$ there exists a unique solution $\hat{u} \in \widehat{\mathcal{D}}_{s}^{1} \subset \widehat{\mathcal{U}}_{s}^{1}$ of problem (3.9) and

$$
\|\hat{u}\|_{\mathcal{U}_{s}^{1}} \leq C e^{\frac{C_{\theta}^{2}}{2 \epsilon}}\|f\|_{\mathcal{U}_{s+\epsilon}^{-1}},
$$

where $C$ is the Poincaré inequality constant that is independent of $\epsilon, s$, and $\theta$.

Proof of (1). From (3.3) we have

$$
\begin{aligned}
a(u, v) & =\int_{D \times \mathcal{S}^{\prime}} \kappa(x, \omega) \nabla u(x, \omega) \nabla v(x, \omega) d x d \mu(\omega) \\
& \leq e^{\frac{C_{\theta}^{2}}{2 \epsilon}} \int_{\mathcal{S}^{\prime}} e^{\frac{\epsilon}{2}|\omega|_{-\theta}^{2}}|u(\cdot, \omega)|_{H_{0}^{1}(D)}|v(\cdot, \omega)|_{H_{0}^{1}(D)} d \mu(\omega) \leq e^{\frac{C_{\theta}^{2}}{2 \epsilon}}|u|_{\mathcal{U}_{s}^{1}}|v|_{\mathcal{U}_{-s+\epsilon}^{1}} .
\end{aligned}
$$


Proof of (2). Given $u \in \widehat{\mathcal{U}}_{s}^{1} \backslash\{0\}$ define

$$
v_{r}(x, \omega):= \begin{cases}u(x, \omega) e^{\left(s+\frac{\epsilon}{2}\right)\|\omega\|_{-\theta}^{2}} & \text { if }\|\omega\|_{-\theta} \leq r \\ 0 & \text { if }\|\omega\|_{-\theta}>r .\end{cases}
$$

Denote $B(r):=\left\{\omega \in \mathcal{S}^{\prime}:\|\omega\|_{-\theta} \leq r\right\}$. From (3.3) we see that

$$
\begin{aligned}
a\left(u, v_{r}\right) & =\int_{D \times B(r)} \kappa(x, \omega)|\nabla u(x, \omega)|^{2} e^{\left(s+\frac{\epsilon}{2}\right)\|\omega\|_{-\theta}^{2}} d x d \mu(\omega) \\
& \leq e^{\frac{C_{\theta}^{2}}{2 \epsilon}} \int_{D \times B(r)}|\nabla u(x, \omega)|^{2} e^{(s+\epsilon)\|\omega\|_{-\theta}^{2}} d x d \mu(\omega) \\
& \leq e^{\frac{C_{\theta}^{2}}{2 \epsilon}} e^{\epsilon r^{2}} \int_{D \times B(r)}|\nabla u(x, \omega)|^{2} e^{s\|\omega\|_{-\theta}^{2}} d x d \mu(\omega) \leq e^{\frac{C_{\theta}^{2}}{2 \epsilon}+\epsilon r^{2}}|u|_{\mathcal{U}_{s}^{1}}^{2}<\infty
\end{aligned}
$$

and therefore, $a\left(u, v_{r}\right)$ is well defined for all $r$. We also have

$$
\begin{aligned}
\left|v_{r}\right|_{\mathcal{U}_{-s-\epsilon}^{1}}^{2} & =\int_{D \times B(r)}|\nabla u(x, \omega)|^{2} e^{2\left(s+\frac{\epsilon}{2}\right)\|\omega\|_{-\theta}^{2}} e^{-(s+\epsilon)\|\omega\|_{-\theta}^{2}} d x d \mu(\omega) \\
& =\int_{D \times B(r)}|\nabla u(x, \omega)|^{2} e^{s\|\omega\|_{-\theta}^{2}} d x d \mu(\omega) \leq|u|_{\mathcal{U}_{s}^{1}}^{2}
\end{aligned}
$$

and using (3.3) we obtain

$$
\begin{aligned}
a\left(u, v_{r}\right) & =\int_{D \times B(r)} \kappa(x, \omega)|\nabla u(x, \omega)|^{2} e^{\left(s+\frac{\epsilon}{2}\right)\|\omega\|_{-\theta}^{2}} d x d \mu(\omega) \\
& \geq e^{-\frac{C_{\theta}^{2}}{2 \epsilon}} \int_{D \times B(r)} e^{-\frac{\epsilon}{2}\|\omega\|_{-\theta}^{2}}|\nabla u(x, \omega)|^{2} e^{\left(s+\frac{\epsilon}{2}\right)\|\omega\|_{-\theta}^{2}} d x d \mu(\omega) \\
& =e^{-\frac{C_{\theta}^{2}}{2 \epsilon}} \int_{D \times B(r)}|\nabla u(x, \omega)|^{2} e^{s\|\omega\|_{-\theta}^{2}} d x d \mu(\omega) .
\end{aligned}
$$

For any arbitrary small $\delta>0$, take $r_{\delta}>0$ sufficiently large such that

$$
\int_{D \times B\left(r_{\delta}\right)}|\nabla u(x, \omega)|^{2} e^{s\|\omega\|_{-\theta}^{2}} d x d \mu(\omega) \geq(1-\delta)|u|_{\mathcal{U}_{s}^{1}}^{2} .
$$

We obtain $a\left(u, v_{r_{\delta}}\right) \geq(1-\delta) e^{-\frac{C_{\theta}^{2}}{2 \epsilon}}|u|_{\mathcal{U}_{s}^{1}}^{2}$ and then

$$
\sup _{v \in \mathcal{U}_{-s-\epsilon}^{1}} \frac{a(u, v)}{|v|_{\mathcal{U}_{-s-\epsilon}^{1}}} \geq \frac{a\left(u, v_{r_{\delta}}\right)}{\left|v_{r_{\delta}}\right|_{\mathcal{U}_{-s-\epsilon}^{1}}^{1}} \geq(1-\delta) e^{-\frac{C_{\theta}^{2}}{2 \epsilon}} \frac{|u|_{\mathcal{U}_{s}^{1}}^{2}}{|u|_{\mathcal{U}_{s}^{1}}^{1}}=(1-\delta) e^{-\frac{C_{\theta}^{2}}{2 \epsilon}}|u|_{\mathcal{U}_{s}^{1}} .
$$

Because $\delta>0$ is arbitrary, we conclude that the inf-sup condition (3.10) holds.

Proof of (3). Given $v \in \widehat{\mathcal{U}}_{-s-\epsilon}^{1} \backslash\{0\}$ we can take $u_{r}$ defined by

$$
u_{r}(x, \omega):= \begin{cases}v(x, \omega) e^{\left(-s-\frac{\epsilon}{2}\right)\|\omega\|_{-\theta}^{2}}, & \|\omega\|_{-\theta} \leq r \\ 0, & \|\omega\|_{-\theta}>r\end{cases}
$$

Note that

$$
\begin{aligned}
\left|u_{r}\right|_{\mathcal{U}_{s+2 \epsilon}^{1}}^{2} & =\int_{D \times B(r)}|\nabla v(x, \omega)|^{2} e^{2\left(-s-\frac{\epsilon}{2}\right)\|\omega\|_{-\theta}^{2}} e^{(s+2 \epsilon)\|\omega\|_{-\theta}^{2}} d x d \mu(\omega) \\
& \leq e^{2 \epsilon r^{2}} \int_{D \times B(r)}|\nabla v(x, \omega)|^{2} e^{(-s-\epsilon)\|\omega\|_{-\theta}^{2}} d x d \mu(\omega) \leq e^{2 \epsilon r^{2}}|v|_{\mathcal{U}_{-s-\epsilon}^{1}}^{2}<\infty
\end{aligned}
$$


By taking $\bar{r}$ large enough we obtain

$$
a\left(u_{\bar{r}}, v\right) \geq e^{-\frac{C_{\theta}^{2}}{2 \epsilon}} \int_{D \times B(\bar{r})} e^{-\frac{\epsilon}{2}\|\omega\|_{-\theta}^{2}}|\nabla v(x, \omega)|^{2} e^{\left(-s-\frac{\epsilon}{2}\right)\|\omega\|_{-\theta}^{2}} d x d \mu(\omega)>0 .
$$

Proof of (4). Let $T_{a}: \widehat{\mathcal{U}}_{s}^{1} \rightarrow \mathcal{U}_{s-\epsilon}^{-1}$ be the linear continuous operator defined by

$$
a(u, v)=\left\langle T_{a} u, v\right\rangle \quad \text { for all } u \in \widehat{\mathcal{U}}_{s}^{1}, \quad v \in \widehat{\mathcal{U}}_{-s+\epsilon}^{1},
$$

and let $\mathcal{R}\left(T_{a}\right)$ be the range of $T_{a}$. Now we show that the subspace $\mathcal{R}\left(T_{a}\right) \cap \mathcal{U}_{s+\epsilon}^{-1}$ is closed in $\mathcal{U}_{s+\epsilon}^{-1}$. Indeed, let $\left\{u_{n}\right\} \subset \widehat{\mathcal{U}}_{s}^{1}$ be a sequence such that the sequence $\left\{T_{a} u_{n}\right\} \subset \mathcal{U}_{s+\epsilon}^{-1}$ converges to $f$ in $\mathcal{U}_{s+\epsilon}^{-1}$. From the inf-sup (3.10), for all integers $m$ and $n$ we have

$$
\begin{aligned}
\left|u_{m}-u_{n}\right|_{\mathcal{U}_{s}^{1}} & \leq e^{\frac{C_{\theta}^{2}}{2 \epsilon}} \sup _{v \in \widehat{\mathcal{U}}_{-s-\epsilon}^{1} \backslash\{0\}} \frac{a\left(u_{m}-u_{n}, v\right)}{|v|_{\mathcal{U}_{-s-\epsilon}^{1}}} \\
& \leq e^{\frac{C_{\theta}^{2}}{2 \epsilon}} \sup _{v \in \widehat{\mathcal{U}}_{-s-\epsilon}^{1} \backslash\{0\}} \frac{\left\langle T_{a}\left(u_{m}-u_{n}\right), v\right\rangle}{|v|_{\mathcal{U}_{-s-\epsilon}^{1}}} \leq e^{\frac{C_{\theta}^{2}}{2 \epsilon}}\left\|T_{a}\left(u_{m}-u_{n}\right)\right\|_{\mathcal{U}_{s+\epsilon}^{-1}},
\end{aligned}
$$

which implies that $\left\{u_{n}\right\}$ is a Cauchy sequence in $\widehat{\mathcal{U}}_{s}^{1}$ and hence has a limit $u \in \widehat{\mathcal{U}}_{s}^{1}$. By continuity we have $T_{a} u=f$, and hence $f \in \mathcal{R}\left(T_{a}\right)$. Since $f \in \mathcal{U}_{s+\epsilon}^{-1}$ we have $f \in \mathcal{R}\left(T_{a}\right) \cap \mathcal{U}_{s+\epsilon}^{-1}$. Hence, we have shown that $\mathcal{R}\left(T_{a}\right) \cap \mathcal{U}_{s+\epsilon}^{-1}$ is closed in $\mathcal{U}_{s+\epsilon}^{-1}$.

Now we show $\mathcal{R}\left(T_{a}\right) \cap \mathcal{U}_{s+\epsilon}^{-1}=\mathcal{U}_{s+\epsilon}^{-1}$. Since $\mathcal{R}\left(T_{a}\right) \cap \mathcal{U}_{s+\epsilon}^{-1}$ is closed in $\mathcal{U}_{s+\epsilon}^{-1}$, assume by contradiction that there exists $v \in\left(\mathcal{U}_{s+\epsilon}^{-1}\right)^{*}=\widehat{\mathcal{U}}_{-s-\epsilon}^{1}$ such that $v \neq 0$ and $\langle f, v\rangle=0$ for all $f \in \mathcal{R}\left(T_{a}\right) \cap \mathcal{U}_{s+\epsilon}^{-1}$. With $u_{\bar{r}}$ introduced in (3), we have from (3.3)

$$
\left\langle T_{a} u_{\bar{r}}, z\right\rangle=a\left(u_{\bar{r}}, z\right) \leq e^{\frac{C_{\theta}^{2}}{2 \epsilon}}\left|u_{\bar{r}}\right|_{\mathcal{U}_{s+2 \epsilon}^{1}}|z|_{\mathcal{U}_{-s-\epsilon}^{1}} \text { for all } z \in \widehat{\mathcal{U}}_{-s-\epsilon}^{1},
$$

which implies that $T_{a} u_{\bar{r}} \in\left(\widehat{\mathcal{U}}_{-s-\epsilon}^{1}\right)^{*}=\mathcal{U}_{s+\epsilon}^{-1}$. Taking $f=T_{a} u_{\bar{r}}$ implies $a\left(u_{\hat{r}}, v\right)=$ $\left\langle T_{a} u_{\bar{r}}, v\right\rangle=0$, and using (3.12) we conclude that $v=0$, which gives a contradiction; hence $\mathcal{R}\left(T_{a}\right) \cap \mathcal{U}_{s+\epsilon}^{-1}=\mathcal{U}_{s+\epsilon}^{-1}$. Thus, the problem (3.9) has a unique solution in $\widehat{\mathcal{U}}_{s}^{1}$ when the right-hand side $f \in \mathcal{U}_{s+\epsilon}^{-1}$, i.e., a unique solution in $\widehat{\mathcal{D}}_{s}^{1} \subset \widehat{\mathcal{U}}_{s}^{1}$. Now (3.11) follows directly from the inf-sup condition (3.10).

Remark 3.2. From Theorem 3.1, when $f \in \mathcal{U}_{0}^{-1}$ then for every $s<0$ (take $\left.\epsilon=-s\right)$ the solution $u \in \widehat{\mathcal{U}}_{s}^{1}$. In order for $u \in \widehat{\mathcal{U}}_{0}^{1}$ we need $f \in \mathcal{U}_{\epsilon}^{-1}$ for some $\epsilon>0$. When the right-hand side $f$ is given by a finite sum of Fourier-Hermite polynomials we have the solution $u \in \widehat{\mathcal{U}}_{s}^{1}$ for every $s$ satisfying $s<\frac{\lambda_{1}^{2 \theta}}{2}$; see Definition 4.1 and Lemma 4.2. We also note that for the case $s=0$ the formulation (3.9) can be formalized with $\hat{u}, \hat{v} \in \widehat{\mathcal{U}}_{0}^{1}$, however, using the inner product $\left(L^{2}\right)_{\epsilon}$.

Remark 3.3. It is easy to see that when $\kappa(x, \omega)=: \rho_{0}+e^{W_{\phi}(x, \omega)}$ and $\rho_{0}>0$, Theorem 3.1 can be slightly modified to establish the well-posedness of the following problem:

$$
\left\{\text { Find } \hat{u} \in \widehat{\mathcal{D}}_{s}^{1} \subset \widehat{\mathcal{U}}_{s}^{1} \text { such that } a(\hat{u}, v)=\langle f, v\rangle \text { for all } v \in \widehat{\mathcal{U}}_{-s}^{1}\right.
$$

with the stabilty $\|\hat{u}\|_{\mathcal{U}_{s}^{1}} \leq \frac{C}{\rho}\|f\|_{\mathcal{U}_{s}^{-1}}$. Here $C$ is the Poincaré inequality constant which is independent of $s$ and $\theta$, and $\widehat{\mathcal{D}}_{s}^{1}$ is the subspace of $u \in \widehat{\mathcal{U}}_{s}^{1}$ such that $T_{a} u \in \mathcal{U}_{s}^{-1}$. 
Additionally, the discrete formulation and the a priori error estimates that we establish in the following sections can also be easily modified to avoid the dependence on $\epsilon$.

Remark 3.4. If $\widetilde{U} \subset \widehat{\mathcal{U}}_{s+\epsilon}^{1} \subset \widehat{\mathcal{U}}_{s}^{1}$ for some $\epsilon>0$, then the pair of spaces $\widetilde{U}$ and $\widetilde{V}$, where $\widetilde{V}:=\left\{u e^{\left(s+\frac{\epsilon}{2}\right)\|\cdot\|_{-\theta}^{2}} ; u \in \widetilde{U}\right\}$, also satisfies the inf-sup condition. This will be useful when constructing finite element spaces in section 5; see Remark 5.1.

4. Characterization of the spaces $\left(L^{2}\right)_{s}$ and $\mathcal{U}_{s}^{m}$. In the following we characterize the space $\left(L^{2}\right)_{s}$ defined in (3.6), and note that this is enough for characterizing the tensor product space $\mathcal{U}_{s}^{m}=H^{m}(D) \otimes\left(L^{2}\right)_{s}$.

We need to consider multi-index of arbitrary length. To simplify the notation, we regard multi-indices as elements of the space $\left(\mathbb{N}_{0}^{\mathbb{N}}\right)_{c}$ of all sequences $\boldsymbol{\alpha}=\left(\alpha_{1}, \alpha_{2}, \ldots\right)$ with elements $\alpha_{j} \in \mathbb{N}_{0}=\mathbb{N} \cup\{0\}$ and with compact support, i.e., with only finitely many $\alpha_{j} \neq 0$. We write $\mathcal{J}=\left(\mathbb{N}_{0}^{\mathbb{N}}\right)_{c}$. Given $\boldsymbol{\alpha} \in \mathcal{J}$ define the order and length of $\boldsymbol{\alpha}$, denoted by $d(\boldsymbol{\alpha})$ and $|\boldsymbol{\alpha}|$, respectively, by

$$
d(\boldsymbol{\alpha}):=\max \left\{j: \alpha_{j} \neq 0\right\} \quad \text { and } \quad|\boldsymbol{\alpha}|:=\alpha_{1}+\alpha_{2}+\cdots+\alpha_{d(\boldsymbol{\alpha})} .
$$

We also introduce the $r$-Hermite polynomials, $h_{r^{2}, n}$, where $r>0$ and $n=0,1,2, \ldots$ These polynomials can be defined by the generating function identity

$$
e^{t x-\frac{1}{2} r^{2} t^{2}}=\sum_{n=0}^{\infty} \frac{t^{n}}{n !} h_{r^{2}, n}(x) .
$$

When $r^{2}=1$ we denote $h_{1, n}$ simply by $h_{n}$. The $r$-Hermite polynomials $h_{r^{2}, n}$ are an orthogonal basis for $L^{2}\left(\mathbb{R}, e^{-\frac{1}{2 r^{2}} x^{2}} d x\right)$ and satisfy $h_{r^{2}, n}(x)=r^{n} h_{1, n}(x / r)$.

For $s<\frac{\lambda_{1}^{2 \theta}}{2}$ define $\sigma_{j}=\sigma_{j}(s):=\left(1-\frac{2 s}{\lambda_{j}^{2 \theta}}\right)^{-\frac{1}{2}}, j=1,2, \ldots$, and for $\boldsymbol{\alpha} \in \mathcal{J}$ let

$$
\sigma^{\boldsymbol{\alpha}}=\sigma^{\boldsymbol{\alpha}}(s):=\prod_{j=1}^{d(\boldsymbol{\alpha})} \sigma_{j}^{\alpha_{j}}(s) \quad \text { and } \quad \sigma_{*}=\sigma_{*}(s):=\int_{\mathcal{S}^{\prime}} e^{s\|\omega\|_{-\theta}^{2}} d \mu(\omega) .
$$

From Lemma 2.2, $\sigma_{*}=\prod_{j=1}^{\infty} \sigma_{j}<\infty$ when $s<\frac{\lambda_{1}^{2 \theta}}{2}$. Now we define the $\sigma(s)$ Fourier-Hermite polynomials.

Definition 4.1. Given $s<\frac{\lambda_{1}^{2 \theta}}{2}, \boldsymbol{\alpha}=\left(\alpha_{1}, \alpha_{2}, \ldots\right) \in \mathcal{J}$, and $\sigma=\sigma(s)=$ $\left(\sigma_{1}, \sigma_{2}, \ldots\right)$, define

$$
H_{\sigma^{2}, \boldsymbol{\alpha}}(\omega):=\frac{1}{\sqrt{\sigma_{*}}} \prod_{j=1}^{d(\boldsymbol{\alpha})} h_{\sigma_{j}^{2}, \alpha_{j}}\left(\left\langle\omega, \eta_{j}\right\rangle\right) \quad \omega \in \mathcal{S}^{\prime} .
$$

We now state the Wiener-Chaos expansion lemma; see Da Prato [11], Hida [19], Holden et al. [21], Hida et al. [20], and Obata [30]. For completeness we include the proof of the orthogonality of the $\sigma(s)$-Fourier-Hermite polynomials.

LEMma 4.2. When $s<\frac{\lambda_{1}^{2 \theta}}{2}$, the $\sigma(s)$-Fourier-Hermite polynomials are orthogonal in $\left(L^{2}\right)_{s}$. Moreover,

$$
\left\|H_{\sigma^{2}(s), \boldsymbol{\alpha}}\right\|_{\left(L^{2}\right)_{s}}^{2}=\boldsymbol{\alpha} ! \sigma(s)^{2 \boldsymbol{\alpha}} .
$$

In addition, every polynomial in $\omega$ belongs to $\left(L^{2}\right)_{s}$, and every $u \in\left(L^{2}\right)_{s}$ can be represented as a Wiener-Chaos expansion,

$$
u=\sum_{\boldsymbol{\alpha} \in \mathcal{J}} u_{\boldsymbol{\alpha}, s} H_{\sigma(s)^{2}, \boldsymbol{\alpha}} \quad \text { with } \quad\|u\|_{\left(L^{2}\right)_{s}}^{2}=\sum_{\boldsymbol{\alpha} \in \mathcal{J}} \boldsymbol{\alpha} ! \sigma(s)^{2 \boldsymbol{\alpha}} u_{\boldsymbol{\alpha}, s}^{2} .
$$


Proof. Take $\boldsymbol{\alpha}, \boldsymbol{\beta} \in \mathcal{J}$ and let $M=\max \{d(\boldsymbol{\alpha}), d(\boldsymbol{\beta})\}$. Note that the random variables

$$
\prod_{j=1}^{M} h_{\sigma_{j}^{2}, \alpha_{j}}\left(\left\langle\cdot, \eta_{j}\right\rangle\right) h_{\sigma_{j}^{2}, \beta_{j}}\left(\left\langle\cdot, \eta_{j}\right\rangle\right) e^{s \lambda_{j}^{-2 \theta}\left\langle\cdot, \eta_{j}\right\rangle} \quad \text { and } \quad \prod_{j=M+1}^{\infty} e^{s \lambda_{j}^{-2 \theta}\left\langle\cdot, \eta_{j}\right\rangle}
$$

are independent (see Remark 2.1). Then

$$
\begin{aligned}
& \frac{1}{\sigma_{*}} \int_{\mathcal{S}^{\prime}} H_{\sigma^{2}, \boldsymbol{\alpha}}(\omega) H_{\sigma^{2} \boldsymbol{\beta}}(\omega) e^{s\|\omega\|_{-\theta}^{2}} d \mu(\omega) \\
= & \int_{\mathcal{S}^{\prime}} \prod_{j=1}^{M} h_{\sigma_{j}^{2}, \alpha_{j}}\left(\left\langle\cdot, \eta_{j}\right\rangle\right) h_{\sigma_{j}^{2}, \beta_{j}}\left(\left\langle\cdot, \eta_{j}\right\rangle\right) \frac{e^{s \lambda_{j}^{-2 \theta}\left\langle\cdot, \eta_{j}\right\rangle}}{\sigma_{j}(s)} d \mu \int_{\mathcal{S}^{\prime}} \prod_{j=M+1}^{\infty} \frac{e^{s \lambda_{j}^{-2 \theta}\left\langle\cdot, \eta_{j}\right\rangle}}{\sigma_{j}(s)} d \mu \\
= & \prod_{j=1}^{M} \sigma_{j}^{2 \alpha_{j}} \alpha_{j} ! \delta_{\alpha_{j}, \beta_{j}}=\boldsymbol{\alpha} ! \sigma^{2 \boldsymbol{\alpha}} \delta_{\boldsymbol{\alpha}, \boldsymbol{\beta}} .
\end{aligned}
$$

In $\left(L^{2}\right)_{s}$ with $s<\frac{\lambda_{1}^{2 \theta}}{2}$ we introduce the system of Hilbert norms

$$
\|u\|_{p ; \rho, s}^{2}:=\sum_{\boldsymbol{\alpha} \in \mathcal{J}} \rho(\boldsymbol{\alpha}, p)^{2} \boldsymbol{\alpha} ! \sigma(s)^{2 \boldsymbol{\alpha}} u_{\boldsymbol{\alpha}, s}^{2},
$$

where $u=\sum_{\boldsymbol{\alpha} \in \mathcal{J}} u_{\boldsymbol{\alpha}, s} H_{\sigma(s)^{2}, \boldsymbol{\alpha}}$. We assume that $\rho(\boldsymbol{\alpha}, q) \geq \rho(\boldsymbol{\alpha}, p)>0$ and $\rho(\boldsymbol{\alpha}, 0)=1$ for all $q>p \geq 0$ and $\boldsymbol{\alpha} \in \mathcal{J}$. Usually, the weights $\rho(\boldsymbol{\alpha}, s)$ are the eigenvalues of some nonnegative operator in $\left(L^{2}\right)_{s}$ with the $\sigma(s)$-Fourier-Hermite polynomials as eigenfunctions; see Benth and Gjerde [4], Holden et al. [21], Hida et al. [20], Obata [30], Kuo [25], Bogachev [7], Cochran, Kuo, and Sengupta [10], and Benth and Theting [5].

For $p>0$ define the spaces $\left(\mathcal{S}_{p}\right)_{\rho, s}$ by

$$
\left(\mathcal{S}_{p}\right)_{\rho, s}:=\left\{v \in\left(L^{2}\right)_{s}:\|v\|_{p ; \rho, s}<\infty\right\} .
$$

For $p<0$ define $\left(\mathcal{S}_{p}\right)_{\rho, s}$ as the dual space of $\left(\mathcal{S}_{-p}\right)_{\rho, s}$. We have $\left(\mathcal{S}_{0}\right)_{\rho, s}=\left(L^{2}\right)_{s}$ and the inclusion $\left(\mathcal{S}_{q}\right)_{\rho, s} \subset\left(\mathcal{S}_{p}\right)_{\rho, s}$ holds for all $q>p$.

Let $N, K \in \mathbb{N}_{0}$ and define

$$
\mathcal{J}^{N, K}:=\{\boldsymbol{\alpha} \in \mathcal{J}: d(\boldsymbol{\alpha}) \leq K \text { and }|\boldsymbol{\alpha}| \leq N\}
$$

and $\mathcal{P}^{N, K}:=\operatorname{span}\left\{H_{\sigma(s)^{2}, \boldsymbol{\alpha}}: \boldsymbol{\alpha} \in \mathcal{J}^{N, K}\right\}=\operatorname{span}\left\{\prod_{j=1}^{d(\boldsymbol{\alpha})}\left\langle\omega, \eta_{j}\right\rangle^{\alpha_{j}}: \boldsymbol{\alpha} \in \mathcal{J}^{N, K}\right\}$; i.e., $\mathcal{P}^{N, K}$ consists of polynomials in $\left\langle\omega, \eta_{1}\right\rangle, \ldots,\left\langle\omega, \eta_{K}\right\rangle$ of total degree at most $N$. Let $Q_{s}^{N, K}$ be the orthogonal projection on $\mathcal{P}^{N, K}$ in the $\left(L^{2}\right)_{s}$-norm. This projection is equivalent to the procedure of truncating the expansion in terms of $\sigma(s)$-FourierHermite polynomials by eliminating the coefficients corresponding to multi-indices outside $\mathcal{J}^{N, K}$. We also define the $\mathbb{R}^{K}$ approximation of $\left(L^{2}\right)_{s}$ by

$$
\mathcal{P}^{K}:=\operatorname{span}\left\{H_{\sigma(s)^{2}, \boldsymbol{\alpha}}: d(\boldsymbol{\alpha}) \leq K\right\},
$$

and denote by $Q_{s}^{K}$ the orthogonal projection on $\mathcal{P}^{K}$ in the $\left(L^{2}\right)_{s}$-norm.

We have the following approximation results.

Lemma 4.3. Assume that $s<\frac{\lambda_{1}^{2 \theta}}{2}$. Then for all $v \in\left(\mathcal{S}_{q}\right)_{\rho, s}$ and $p<q$ we have

$$
\left\|v-Q_{s}^{N, K} v\right\|_{p ; \rho, s}^{2} \leq M_{1}^{2}\left\|Q_{s}^{K} v-Q_{s}^{N, K} v\right\|_{q ; \rho, s}^{2}+M_{2}^{2}\left\|v-Q_{s}^{K} v\right\|_{q ; \rho, s}^{2}
$$


with

$$
M_{1}=M_{1}(\rho, p, q):=\max _{d(\boldsymbol{\alpha}) \leq K,|\boldsymbol{\alpha}|>N} \frac{\rho(\boldsymbol{\alpha}, p)}{\rho(\boldsymbol{\alpha}, q)}
$$

and

$$
M_{2}=M_{2}(\rho, p, q):=\max _{d(\boldsymbol{\alpha})>K} \frac{\rho(\boldsymbol{\alpha}, p)}{\rho(\boldsymbol{\alpha}, q)} .
$$

Proof. Fix $s<\frac{\lambda_{1}^{2 \theta}}{2}$ and note that $Q_{s}^{N, K} v=\sum_{\boldsymbol{\alpha} \in \mathcal{J}^{N, K}} v_{\boldsymbol{\alpha}, s} H_{\sigma^{2}, \boldsymbol{\alpha}}$. Then recalling the definition of $\mathcal{J}^{N, K}$ in (4.6) we see that

$$
\begin{aligned}
& \left\|v-Q_{s}^{N, K} v\right\|_{p ; \rho, s}^{2}=\sum_{\boldsymbol{\alpha} \notin \mathcal{J}^{N, K}} \rho(\boldsymbol{\alpha}, p)^{2} \boldsymbol{\alpha} ! \sigma(s)^{2 \boldsymbol{\alpha}} v_{\boldsymbol{\alpha}, s}^{2} \\
= & \sum_{\boldsymbol{\alpha} \notin \mathcal{J}^{N, K}} \rho(\boldsymbol{\alpha}, q)^{2} \boldsymbol{\alpha} ! \sigma(s)^{2 \boldsymbol{\alpha}} v_{\boldsymbol{\alpha}, s}^{2} \frac{\rho(\boldsymbol{\alpha}, p)^{2}}{\rho(\boldsymbol{\alpha}, q)^{2}} \\
= & \sum_{d(\boldsymbol{\alpha}) \leq K,|\boldsymbol{\alpha}|>N} \rho(\boldsymbol{\alpha}, q)^{2} \boldsymbol{\alpha} ! \sigma(s)^{2 \boldsymbol{\alpha}} v_{\boldsymbol{\alpha}, s}^{2} \frac{\rho(\boldsymbol{\alpha}, p)^{2}}{\rho(\boldsymbol{\alpha}, q)^{2}}+\sum_{d(\boldsymbol{\alpha})>K} \rho(\boldsymbol{\alpha}, q)^{2} \boldsymbol{\alpha} ! \sigma(s)^{2 \boldsymbol{\alpha}} v_{\boldsymbol{\alpha}, s}^{2} \frac{\rho(\boldsymbol{\alpha}, p)^{2}}{\rho(\boldsymbol{\alpha}, q)^{2}} \\
\leq & \left(\max _{d(\boldsymbol{\alpha}) \leq K,|\boldsymbol{\alpha}|>N} \frac{\rho(\boldsymbol{\alpha}, p)^{2}}{\rho(\boldsymbol{\alpha}, q)^{2}}\right)\left\|Q_{s}^{K} v-Q_{s}^{N, K} v\right\|_{q ; \rho, s}^{2}+\left(\max _{d(\boldsymbol{\alpha})>K} \frac{\rho(\boldsymbol{\alpha}, p)^{2}}{\rho(\boldsymbol{\alpha}, q)^{2}}\right)\left\|v-Q_{s}^{K} v\right\|_{q ; \rho, s}^{2} \\
\leq & M_{1}^{2}\left\|Q_{s}^{K} v-Q_{s}^{N, K} v\right\|_{q ; \rho, s}^{2}+M_{2}^{2}\left\|v-Q_{s}^{K} v\right\|_{q ; \rho, s}^{2},
\end{aligned}
$$

where $M_{1}$ and $M_{2}$ are defined in (4.7) and (4.8), respectively.

Corollary 4.4. Assume that $s<\frac{\lambda_{1}^{2 \theta}}{2}$. For all $v \in\left(\mathcal{S}_{q}\right)_{\rho, s}$ and $p<q$ we have

$$
\left\|v-Q_{s}^{N, K} v\right\|_{p ; \rho, s} \leq \max \left\{M_{1}, M_{2}\right\}\|v\|_{q ; \rho, s}
$$

with $M_{1}$ and $M_{2}$ defined in (4.7) and (4.8), respectively.

If $v \in\left(\mathcal{S}_{q}\right)_{\rho, s}$ is of finite dimensional noise type, i.e., if $v=\sum_{d(\boldsymbol{\alpha}) \leq K} v_{\boldsymbol{\alpha}, s} H_{\sigma(s)^{2}, \boldsymbol{\alpha}}(\omega)$, then for all $p<q$

$$
\left\|v-Q_{s}^{N, K} v\right\|_{p ; \rho, s} \leq M_{1}(\rho, p, q)\|v\|_{q ; \rho, s} .
$$

Proof. From (4.4) and the definition of the $\left(L^{2}\right)_{s}$ orthogonal projections $Q_{s}^{K}$ and $Q_{s}^{N, K}$, we see that

$$
\left\|Q_{s}^{K} v-Q_{s}^{N, K} v\right\|_{q ; \rho, s}^{2}+\left\|v-Q_{s}^{K} v\right\|_{q ; \rho, s}^{2}=\left\|v-Q_{s}^{N, K} v\right\|_{q ; \rho, s}^{2} \leq\|v\|_{q ; \rho, s}^{2},
$$

which together with Lemma 4.3 give the first inequality. The second inequality follows from Lemma 4.3 after noting that if $v$ is of finite dimensional noise type, then $Q_{s}^{K} v=$ $v$.

Several examples of weights $\rho(\boldsymbol{\alpha}, p)$ can be considered. We next mention two examples.

Example 4.5 (see Cao [9], Benth and Gjerde [4], Holden et al. [21], Kuo [25], and Obata [30]). Take $\nu \in[0,1)$ and

$$
\rho(\boldsymbol{\alpha}, p)^{2}=(\boldsymbol{\alpha} !)^{\nu} \lambda^{2 p \boldsymbol{\alpha}}, \quad \boldsymbol{\alpha} \in \mathcal{J} .
$$


Here we use the notation $\lambda^{\boldsymbol{\beta}}=\prod_{j=1}^{d(\boldsymbol{\beta})} \lambda_{j}^{\beta_{j}}$ for any $\boldsymbol{\beta} \in \mathcal{J}$. Note that we can write

$$
\|u\|_{p ; \rho, s}^{2}=|| \Gamma_{\otimes, \nu}(A)^{p} u \|_{\left(L^{2}\right)_{s}}^{2}=\int_{\mathcal{S}^{\prime}}\left|\Gamma_{\otimes, \nu}(A)^{p} u(\omega)\right|^{2} e^{s\|\omega\|_{-\theta}^{2}} d \mu(\omega),
$$

where $\Gamma_{\otimes, \nu}(A)$ is the operator defined by $\Gamma_{\otimes, \nu}(A) H_{\sigma^{2}, \boldsymbol{\alpha}}=(\alpha !)^{\nu} \lambda^{\boldsymbol{\alpha}} H_{\sigma^{2}, \boldsymbol{\alpha}}$. Note also that $\Gamma_{\otimes, 0}\left(A^{p}\right)=\Gamma_{\otimes, 0}(A)^{p}$. In the case of $\nu=0$ and $s=0, \Gamma_{\otimes, 0}(A)$ is called the Second Quantization of $A$; see Hida et al. [20].

Corollary 4.6. Assume that $s<\frac{\lambda_{1}^{2 \theta}}{2}$ and consider the weights $\rho$ defined in (4.9). Then for every $p<q$ we have

$$
\left\|v-Q_{s}^{N, K} v\right\|_{p ; \rho, s} \leq \max \left\{\frac{1}{\lambda_{1}^{N+1}}, \frac{1}{\lambda_{K+1}}\right\}^{q-p}\|v\|_{q ; \rho, s} .
$$

Proof. Recalling that $1<\lambda_{1} \leq \lambda_{2} \leq \cdots$, we see that for all $q>p$

$$
M_{1}(\rho, p, q)=\max _{d(\boldsymbol{\alpha}) \leq K,|\boldsymbol{\alpha}|>N} \prod_{i=1}^{d(\boldsymbol{\alpha})} \frac{1}{\lambda_{i}^{\alpha_{i}(q-p)}}=\frac{1}{\lambda_{1}^{(N+1)(q-p)}}
$$

and

$$
M_{2}(\rho, p, q)=\max _{d(\boldsymbol{\alpha})>K} \prod_{i=1}^{d(\boldsymbol{\alpha})} \frac{1}{\lambda_{i}^{\alpha_{i}(q-p)}}=\frac{1}{\lambda_{K+1}^{(q-p)}},
$$

and the lemma follows.

Remark 4.7. We note that Corollary 4.6 is valid for any choice of the sequence $\left\{\lambda_{j}\right\}_{j=1}^{\infty}$ with $1<\lambda_{1} \leq \lambda_{2} \leq \cdots$ such that $\sum_{j=1}^{\infty} \lambda_{j}^{-2 \theta}<\infty$; see section 7.1.1. For instance, Corollary 4.6 applied to the sequence $\left\{\lambda_{j}=2 j\right\}_{j=1}^{\infty}$ with $\theta=1(s<2)$ gives for all $p \in \mathbb{R}$ and $t>0$,

$$
\left\|v-Q_{s}^{N, K} v\right\|_{p ; \rho, s} \leq \frac{1}{2^{t}} \max \left\{\frac{1}{2^{t N}}, \frac{1}{(K+1)^{t}}\right\}\|v\|_{p+t ; \rho, s} .
$$

Benth and Gjerde [4] and Cao [9] consider the norms of Example 4.5 with weights $\rho$ defined in (4.9) for the special case $s=0$ and the sequence $\left\{\lambda_{j}=2 j\right\}_{j=1}^{\infty}$. They derive approximation estimates valid only for $p<0$ and $t>1$. Using our notation, Theorem 2 in Cao [9], which substantially improves the result of Benth and Gjerde [4], reads as follows: Let $p<0$ and assume that $t>1$. Then for any $v \in\left(\mathcal{S}_{p+t}\right)_{\rho, 0}$

$$
\left\|v-Q_{s}^{N, K} v\right\|_{p ; \rho, 0} \leq \sqrt{B(t) \frac{1}{2^{t N}}+A(t) \frac{1}{K^{t-1}}}\|v\|_{p+t ; \rho, 0},
$$

where

$$
A(t)=e^{\frac{2}{t-1}} \frac{t}{t-1} \quad \text { and } \quad B(t)=e^{\frac{1}{2^{t-1}(t-1)}} \frac{1}{2^{t}(t-1)} .
$$

It is easy to see that

$$
\frac{1}{2^{t}} \max \left\{\frac{1}{2^{t N}}, \frac{1}{(K+1)^{t}}\right\} \leq \frac{1}{2^{t} \sqrt{B(t)}} \sqrt{B(t) \frac{1}{2^{t N}}+A(t) \frac{1}{K^{t-1}}}
$$


and then our estimate is sharper than the one given in [9] and, moreover, is valid for all $p \in \mathbb{R}$ and $t>0$.

Example 4.8 (see Bogachev [7], Da Prato [11], Hida et al. [20], and Shigekawa [34]). Given a multi-index $\boldsymbol{\alpha}$ we denote $\langle\boldsymbol{\alpha}, \lambda\rangle:=\sum_{i=1}^{d(\boldsymbol{\alpha})} \alpha_{i} \lambda_{i}$. As an alternative to the weights $\rho$ introduced in Example 4.5 we can define

$$
\rho(\boldsymbol{\alpha}, p)^{2}=1+\langle\boldsymbol{\alpha}, \lambda\rangle^{2 p}, \quad p>0, \text { and } \rho(\boldsymbol{\alpha}, 0)=1, \quad \boldsymbol{\alpha} \in \mathcal{J} .
$$

In this case we can write

$$
\begin{aligned}
\|u\|_{p ; \rho, s}^{2} & =\|u\|_{\left(L^{2}\right)_{s}}^{2}+\left\|\Gamma_{\oplus}(A)^{p} u\right\|_{\left(L^{2}\right)_{s}}^{2} \\
& =\int_{\mathcal{S}^{\prime}}\left(|u(\omega)|^{2}+\left|\Gamma_{\oplus}(A)^{p} u(\omega)\right|^{2}\right) e^{s\|\omega\|_{-\theta}^{2}} d \mu(\omega),
\end{aligned}
$$

where $\Gamma_{\oplus}(A)$ is the operator defined by $\Gamma_{\oplus}(A) H_{\sigma^{2}, \boldsymbol{\alpha}}=\langle\boldsymbol{\alpha}, \lambda\rangle H_{\sigma^{2}, \boldsymbol{\alpha}}$. Note also that in this case $\Gamma\left(A^{p}\right) \neq \Gamma(A)^{p}$. It is easy to see that $\left\|\Gamma_{\oplus}(A)^{p} \cdot\right\|_{\left(L^{2}\right)_{s}}^{2}$ is a norm in the space of function in $\left(L^{2}\right)_{s}$ with $u_{0}=0$ in its $\sigma(s)$-Fourier-Hermite expansion. This seminorm can be computed using directional derivatives in the $\omega$ variable; see Da Prato [11].

The proof of the following result is similar to the proof of Corollary 4.6.

Corollary 4.9. Assume that $s<\frac{\lambda_{1}^{2 \theta}}{2}$ and consider $\rho$ defined in (4.11). Then for every $p<q$ we have

$$
\left\|v-Q_{s}^{N, K} v\right\|_{p ; \rho, s} \leq \max \left\{\frac{1}{1+(N+1) \lambda_{1}}, \frac{1}{1+\lambda_{K+1}}\right\}^{q-p}\|v\|_{q ; \rho, s} .
$$

In this section we have only considered results associated with $\left(L^{2}\right)_{s}$. The corresponding tensor product norm for $u \in \mathcal{U}_{s}^{m}:=H^{m}(D) \otimes\left(L^{2}\right)_{s}$ with $s<\frac{\lambda_{1}^{2 \theta}}{2}$ is given by

$$
\|u\|_{\mathcal{U}_{s}^{m}}^{2}=\sum_{\boldsymbol{\alpha} \in \mathcal{J}} \boldsymbol{\alpha} ! \sigma(s)^{2 \boldsymbol{\alpha}}\left\|u_{\boldsymbol{\alpha}, s}\right\|_{H^{m}(D)}^{2}
$$

where $u=\sum_{\boldsymbol{\alpha} \in \mathcal{J}} u_{\boldsymbol{\alpha}, s} H_{\sigma(s)^{2}, \boldsymbol{\alpha}}$ with $u_{\boldsymbol{\alpha}, s} \in H^{m}(D)$ for all $\boldsymbol{\alpha} \in \mathcal{J}$.

Norms $\|\cdot\|_{p ; \rho, s}$ and spaces $\left(\mathcal{S}_{p}\right)_{\rho, s} \subset\left(L^{2}\right)_{s}$ defined in (4.4) and (4.5) can also be extended to tensor products. The corresponding norms for the tensor product spaces $\mathcal{U}_{p ; \rho, s}^{m}:=H^{m}(D) \otimes\left(\mathcal{S}_{p}\right)_{\rho, s}$ are defined by

$$
\|u\|_{\mathcal{U}_{p ; \rho, s}^{m}}^{2}:=\sum_{\boldsymbol{\alpha} \in \mathcal{J}} \rho(\boldsymbol{\alpha}, p)^{2} \boldsymbol{\alpha} ! \sigma(s)^{2 \boldsymbol{\alpha}}\left\|u_{\boldsymbol{\alpha}, s}\right\|_{H^{m}(D)}^{2},
$$

and the seminorm is

$$
|u|_{\mathcal{U}_{p ; \rho, s}^{m}}^{2}:=\sum_{\boldsymbol{\alpha} \in \mathcal{J}} \rho(\boldsymbol{\alpha}, p)^{2} \boldsymbol{\alpha} ! \sigma(s)^{2 \boldsymbol{\alpha}}\left|u_{\boldsymbol{\alpha}, s}\right|_{H^{m}(D)}^{2} .
$$

We have the following important remark.

Remark 4.10. Lemma 4.3 and Corollaries 4.4, 4.6, and 4.9 extend trivially to the tensor product norm and seminorm defined in (4.12) and (4.13). 
5. The Galerkin approximation and a priori error estimates. Recall that when $s<\frac{\lambda_{1}^{2 \theta}}{2}$, polynomials in $\omega$ belong to $\left(L^{2}\right)_{s}$. Let $X_{0}^{h}(D) \subset H_{0}^{1}(D)$ be the finite element space of piecewise linear and continuous functions with respect to a triangulation of $D$. For $N, K \in \mathbb{N}_{0}$, and $h>0$ define the following discrete spaces:

$$
\mathcal{X}_{s}^{N, K, h}:=X_{0}^{h}(D) \otimes \mathcal{P}^{N, K} \subset \widehat{\mathcal{U}}_{s}^{1} \subset \mathcal{U}_{s}^{1}
$$

and

$$
\mathcal{Y}_{s}^{N, K, h}:=\left\{v: v(x, \omega)=\tilde{v}(x, \omega) e^{\left(s+\frac{\epsilon}{2}\right)\left\|\Pi_{K} \omega\right\|_{-\theta}^{2}}, \tilde{v} \in \mathcal{X}_{s}^{N, K, h}\right\} \subset \widehat{\mathcal{U}}_{-(s+\epsilon)}^{1},
$$

where $\Pi_{K}$ is the (H-orthogonal) projection on the $\operatorname{span}\left\{\eta_{1}, \ldots, \eta_{K}\right\}$ defined by

$$
\Pi_{K} \omega:=\sum_{j=1}^{K}\left\langle\omega \eta_{j}\right\rangle \eta_{j} \text { for all } \omega \in \mathcal{S}^{\prime}
$$

Remark 5.1. Note that we have defined $\mathcal{Y}_{s}^{N, K, h}$ in (5.2) by multiplying $\tilde{v}(x, \omega)$ by the weight $e^{\left(s+\frac{\epsilon}{2}\right)\left\|\Pi_{K} \omega\right\|_{-\theta}^{2}}$ rather than by $e^{\left(s+\frac{\epsilon}{2}\right)\|\omega\|_{-\theta}^{2}}$ (see Remark 3.4). This is done in order to avoid computations of infinite series when assembling the resulting linear system; see section 6 . We note also that Remark 3.4 would require the assumption $s+\epsilon<\lambda_{1}^{2 \theta} / 2$ in order to establish the discrete inf-sup condition.

The discrete version of problem (3.9) is introduced as follows:

(5.4) $\left\{\right.$ Find $\hat{u}_{s}^{N, K, h} \in \mathcal{X}_{s}^{N, K, h}$ such that $a\left(\hat{u}_{s}^{N, K, h}, v\right)=\langle f, v\rangle$ for all $v \in \mathcal{Y}_{s}^{N, K, h}$.

Remark 5.2. Observe that the above Galerkin approximation $\hat{u}_{s}^{N, K, h}$ satisfies a variational equation with the original permeability $\kappa$ defined in (3.2).

Note that the functions in $\mathcal{X}_{s}^{N, K, h}$ depend only on the $\left\langle\omega, \eta_{j}\right\rangle, j=1, \ldots, K$, and not on the $\left\langle\omega, \eta_{j}\right\rangle, j=K+1, \ldots$. Therefore, for all $u \in \mathcal{X}_{s}^{N, K, h}$ and $v \in \mathcal{Y}_{s}^{N, K, h}$, i.e., $v(x, \omega)=\tilde{v}(x, \omega) e^{\left(s+\frac{\epsilon}{2}\right)\left\|\Pi_{K} \omega\right\|_{-\theta}^{2}}$ with $\tilde{v} \in \mathcal{X}_{s}^{N, K, h}$ and $\Pi_{K}$ defined in (5.3), we have

$$
\begin{aligned}
& a(u, v)=\int_{D \times \mathcal{S}^{\prime}} e^{\left\langle\omega, \phi_{x}\right\rangle} \nabla u(x, \omega) \nabla \tilde{v}(x, \omega) e^{\left(s+\frac{\epsilon}{2}\right)\left\|\Pi_{K} \omega\right\|_{-\theta}^{2}} d x d \mu(\omega) \\
= & \int_{D \times \mathcal{S}^{\prime}} e^{\sum_{j=1}^{\infty}\left(\phi_{x}, \eta_{j}\right)\left\langle\omega, \eta_{j}\right\rangle} \nabla u(x, \omega) \nabla \tilde{v}(x, \omega) e^{\left(s+\frac{\epsilon}{2}\right)\left\|\Pi_{K} \omega\right\|_{-\theta}^{2}} d x d \mu(\omega) \\
(5.5)= & \int_{D} e^{\frac{1}{2} \sum_{j=K+1}^{\infty} a_{j}(x)^{2}} \int_{\mathcal{S}^{\prime}} e^{\sum_{j=1}^{K} a_{j}(x)\left\langle\cdot, \eta_{j}\right\rangle} \nabla u \nabla \tilde{v} e^{\left(s+\frac{\epsilon}{2}\right)\left\|\Pi_{K} \cdot\right\|_{-\theta}^{2}} d \mu(\omega) d x \\
= & \int_{D} e^{\frac{1}{2}\left(\left\|\phi_{x}\right\|_{H}^{2}-\sum_{j=1}^{K} a_{j}(x)^{2}\right)} \int_{\mathcal{S}^{\prime}} e^{\sum_{j=1}^{K} a_{j}(x)\left\langle\cdot, \eta_{j}\right\rangle} \nabla u \nabla \tilde{v} e^{\left(s+\frac{\epsilon}{2}\right)\left\|\Pi_{K} \cdot\right\|_{-\theta}^{2}} d \mu(\omega) d x,
\end{aligned}
$$

where we have used the formula $\int_{\mathbb{R}} e^{a_{j}(x) y_{j}} \frac{1}{\sqrt{2 \pi}} e^{-\frac{y_{j}^{2}}{2}} d y_{j}=e^{\frac{a_{j}(x)^{2}}{2}}$ and the notation

$$
a_{j}(x):=\left(\phi_{x}, \eta_{j}\right)_{H}
$$

for $j=1, \ldots$ We have the following result.

LemMa 5.3. Let $\epsilon>0$ and $s \in \mathbb{R}$ such that $s<\frac{\lambda_{1}^{2 \theta}}{2}$ and $-s-\epsilon<\frac{\lambda_{K+1}^{2 \theta}}{2}$. The bilinear form " $a$ " and the spaces $\left(\mathcal{X}_{s}^{N, K, h}, \mathcal{Y}_{s}^{N, K, h}\right)$ satisfy the following discrete inf-sup condition:

$$
\inf _{u \in \mathcal{X}_{s}^{N, K, h} \backslash\{0\}} \sup _{v \in \mathcal{Y}_{s}^{N, K, h} \backslash\{0\}} \frac{a(u, v)}{|u|_{\mathcal{U}_{s}^{1}}|v|_{\mathcal{U}_{-(s+\epsilon)}^{1}}} \geq \frac{e^{-\frac{C_{\theta}^{2}}{2 \epsilon}}}{\prod_{j=K+1}^{\infty} \sigma_{j}(-s-\epsilon)} .
$$



and

Proof. Let $u \in \mathcal{X}_{s}^{N, K, h} \backslash\{0\}$. If $v(x, \omega):=u(x, \omega) e^{\left(s+\frac{\epsilon}{2}\right)\left\|\Pi_{K} \omega\right\|_{-\theta}^{2}}$, then $v \in \mathcal{Y}_{s}^{N, K, h}$

$$
\begin{aligned}
& |v|_{\mathcal{U}_{-(s+\epsilon)}^{1}}^{2}=\int_{D \times \mathcal{S}^{\prime}}|\nabla u(x, \omega)|^{2} e^{2\left(s+\frac{\epsilon}{2}\right)\left\|\Pi_{K} \omega\right\|_{-\theta}^{2}} e^{-(s+\epsilon)\|\omega\|_{-\theta}^{2}} d x d \mu(\omega) \\
& \quad=\int_{D \times \mathcal{S}^{\prime}}|\nabla u(x, \omega)|^{2} e^{2\left(s+\frac{\epsilon}{2}\right)\left\|\Pi_{K} \omega\right\|_{-\theta}^{2}} e^{-(s+\epsilon)\left(\left\|\Pi_{K} \omega\right\|_{-\theta}^{2}+\left\|\left(I-\Pi_{K}\right) \omega\right\|_{-\theta}^{2}\right)} d x d \mu(\omega) .
\end{aligned}
$$

As in Lemma 2.2, for $-(s+\epsilon)<\frac{\lambda_{K+1}^{2 \theta}}{2}$ we have

$$
\int_{\mathcal{S}^{\prime}} e^{-(s+\epsilon)\left\|\left(I-\Pi_{K}\right) \omega\right\|_{-\theta}^{2}} d \mu(\omega)=\prod_{j=K+1}^{\infty} \sigma_{j}(-s-\epsilon)<\infty .
$$

Analogous computation holds for $\int_{S^{\prime}} e^{s\left\|\left(I-\Pi_{K}\right) \omega\right\|_{-\theta}^{2}} d \mu(\omega)$ when $s<\frac{\lambda_{K+1}^{2 \theta}}{2}$. Then,

$$
\begin{aligned}
|v|_{\mathcal{U}_{-(s+\epsilon)}^{1}}^{2} & =\prod_{j=K+1}^{\infty} \sigma_{j}(-s-\epsilon) \int_{D \times \mathcal{S}^{\prime}}|\nabla u(x, \omega)|^{2} e^{s\left\|\Pi_{K} \omega\right\|_{-\theta}^{2}} d x d \mu(\omega) \\
& =\frac{\prod_{j=K+1}^{\infty} \sigma_{j}(-s-\epsilon)}{\prod_{j=K+1}^{\infty} \sigma_{j}(s)} \int_{D \times \mathcal{S}^{\prime}}|\nabla u(x, \omega)|^{2} e^{s\|\omega\|_{-\theta}^{2}} d x d \mu(\omega) \\
& =\frac{\prod_{j=K+1}^{\infty} \sigma_{j}(-s-\epsilon)}{\prod_{j=K+1}^{\infty} \sigma_{j}(s)}|u|_{\mathcal{U}_{s}^{1}}^{2},
\end{aligned}
$$

and from (5.5) and the fact that $e^{\frac{1}{2} \sum_{j=K+1}^{\infty} a_{j}(x)^{2}} \geq 1$, we have

$$
\begin{aligned}
a(u, v) & \geq \int_{D \times \mathcal{S}^{\prime}} e^{\sum_{j=1}^{K} a_{j}(x)\left\langle\omega, \eta_{j}\right\rangle}|\nabla u(x, \omega)|^{2} e^{\left(s+\frac{\epsilon}{2}\right)\left\|\Pi_{K} \omega\right\|_{-\theta}^{2}} d x d \mu(\omega) \\
& \geq e^{-\frac{C_{\theta}^{2}}{2 \epsilon}} \int_{D \times \mathcal{S}^{\prime}} e^{-\frac{\epsilon}{2}\left|\Pi_{K} \omega\right|_{-\theta}^{2}}|\nabla u(x, \omega)|^{2} e^{\left(s+\frac{\epsilon}{2}\right)\left\|\Pi_{K} \omega\right\|_{-\theta}^{2}} d x d \mu(\omega) \\
& =e^{-\frac{C_{\theta}^{2}}{2 \epsilon}} \int_{D \times \mathcal{S}^{\prime}}|\nabla u(x, \omega)|^{2} e^{s\left\|\Pi_{K} \omega\right\|_{-\theta}^{2}} d x d \mu(\omega)=\frac{1}{\prod_{j=K+1}^{\infty} \sigma_{j}(s)} e^{-\frac{C_{\theta}^{2}}{2 \epsilon}}|u|_{\mathcal{U}_{s}^{1}}^{2} .
\end{aligned}
$$

Hence,

$$
\frac{a(u, v)}{|v|_{\mathcal{U}_{-(s+\epsilon)}^{1}}} \geq \frac{\frac{1}{\prod_{j=K+1}^{\infty} \sigma_{j}(s)}}{\frac{\prod_{j=K+1}^{\infty} \sigma_{j}(-s-\epsilon)}{\prod_{j=K+1}^{\infty} \sigma_{j}(s)}} e^{-\frac{C_{\theta}^{2}}{2 \epsilon}} \frac{|u|_{\mathcal{U}_{s}^{1}}^{2}}{|u|_{\mathcal{U}_{s}^{1}}^{1}} \geq \frac{e^{-\frac{C_{\theta}^{2}}{2 \epsilon}}}{\prod_{j=K+1}^{\infty} \sigma_{j}(-s-\epsilon)}|u|_{\mathcal{U}_{s}^{1}}
$$

We conclude that the discrete inf-sup condition holds.

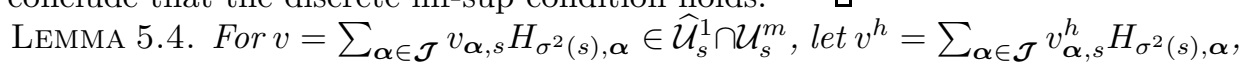
where $v_{\boldsymbol{\alpha}, s}^{h}$ is the Clement finite element interpolation of $v_{\boldsymbol{\alpha}, s}$ on the space $X_{0}^{h}(D)$. Then

$$
\left\|v-v^{h}\right\|_{\mathcal{U}_{s}^{1}} \leq \hat{C} h^{\ell-1}\|v\|_{\mathcal{U}_{s}^{\ell}}, \quad \ell=1,2,
$$

where the constant $\hat{C}$ is independent of $s$ and $h$.

Using the tensor product norm (4.12) we can easily deduce the following a priori error estimates. 
Lemma 5.5. Let $s \in \mathbb{R}$ and $\epsilon>0$ such that $s+2 \epsilon<\frac{\lambda_{1}^{2 \theta}}{2}$ and $-s-\epsilon<\frac{\lambda_{K+1}^{2 \theta}}{2}$. Then the following estimate holds:

$$
\left|\hat{u}-\hat{u}_{s}^{N, K, h}\right|_{\mathcal{U}_{s}^{1}} \leq\left(1+e^{\frac{C_{\theta}^{2}}{\epsilon}} \prod_{j=K+1}^{\infty} \sigma_{j}(-s-\epsilon)\right) \inf _{z \in \mathcal{X}_{s}^{N, K, h}}|\hat{u}-z|_{\mathcal{U}_{s+2 \epsilon}^{1}} .
$$

Moreover, for all $q>0$

$$
\left|\hat{u}-\hat{u}_{s}^{N, K, h}\right|_{\mathcal{U}_{s}^{1}} \leq C_{*}\left\{\max \left\{M_{1}(\rho, 0, q), M_{2}(\rho, 0, q)\right\}|\hat{u}|_{\mathcal{U}_{q ; \rho, s+2 \epsilon}^{1}}+\hat{C} h^{\ell-1}\|\hat{u}\|_{\mathcal{U}_{s+2 \epsilon}^{\ell}}\right\},
$$

where $C_{*}=C_{*}(s, \epsilon)=1+e^{\frac{C_{\theta}^{2}}{\epsilon}} \prod_{j=K+1}^{\infty} \sigma_{j}(-s-\epsilon), M_{1}$ and $M_{2}$ are defined in Corollary 4.4 , and $\hat{C}$ is the constant of Lemma 5.4 .

Proof. First note that for all $v \in \mathcal{Y}_{s}^{N, K, h}$ and $z \in \mathcal{X}_{s}^{N, K, h}$

$$
a\left(\hat{u}_{s}^{N, K, h}-z, v\right)=a(\hat{u}-z, v) .
$$

Using the continuity (2) in Theorem 3.1, with $s+2 \epsilon$ instead of $s$, we obtain

$$
a\left(\hat{u}_{s}^{N, K, h}-z, v\right) \leq e^{\frac{C_{\theta}^{2}}{2 \epsilon}}|\hat{u}-z|_{\mathcal{U}_{s+2 \epsilon}^{1}}|v|_{\mathcal{U}_{-s-\epsilon}^{1}} .
$$

From the discrete inf-sup of Lemma 5.3 we have

$$
\begin{aligned}
\left|\hat{u}-\hat{u}_{s}^{N, K, h}\right|_{\mathcal{U}_{s}^{1}} \leq|\hat{u}-z|_{\mathcal{U}_{s}^{1}}+\left|\hat{u}_{s}^{N, K, h}-z\right|_{\mathcal{U}_{s}^{1}} & \leq|\hat{u}-z|_{\mathcal{U}_{s}^{1}}+e^{\frac{C_{\theta}^{2}}{2 \epsilon}} \prod_{j=K+1}^{\infty} \sigma_{j}(-s-\epsilon) \sup _{v \in \mathcal{Y}_{s}^{N, K, h} \backslash\{0\}} \frac{a\left(\hat{u}_{s}^{N, K, h}-z, v\right)}{|v|_{\mathcal{U}_{-s-\epsilon}^{1}}} \\
& \leq|\hat{u}-z|_{\mathcal{U}_{s}^{1}}+e^{\frac{C_{\theta}^{2}}{2 \epsilon}} e^{\frac{C_{\theta}^{2}}{2 \epsilon}} \prod_{j=K+1}^{\infty} \sigma_{j}(-s-\epsilon)|\hat{u}-z|_{\mathcal{U}_{s+2 \epsilon}^{1}} \\
& \leq\left(1+e^{\frac{C_{\theta}^{2}}{\epsilon}} \prod_{j=K+1}^{\infty} \sigma_{j}(-s-\epsilon)\right)|\hat{u}-z|_{\mathcal{U}_{s+2 \epsilon}^{1}},
\end{aligned}
$$

which gives (5.8). Now we bound the second term of (5.8). This can be done as follows: take the polynomial $z=\zeta_{N, K}^{h} \in \mathcal{X}_{s}^{N, K, h}$, where $\zeta_{N, K}=\left(I d \otimes Q_{s+2 \epsilon}^{N, K}\right) \hat{u}$. Note that polynomials in $\omega$ are in $\left(L^{2}\right)_{s+2 \epsilon}$ since $s+2 \epsilon<\frac{\lambda_{1}^{2 \theta}}{2}$; therefore, $\zeta_{N, K} \in \widehat{\mathcal{U}}_{s+2 \epsilon}^{1}$ is well defined. We have

$$
\left|\hat{u}-\zeta_{N, K}^{h}\right|_{\mathcal{U}_{s+2 \epsilon}^{1}} \leq\left|\hat{u}-\zeta_{N, K}\right|_{\mathcal{U}_{s+2 \epsilon}^{1}}+\left|\zeta_{N, K}-\zeta_{N, K}^{h}\right|_{\mathcal{U}_{s+2 \epsilon}^{1}} .
$$

Apply Corollary 4.4 (see Remark 4.10) with $p=0$ and $q>0$ to obtain

$$
\begin{aligned}
\left|\hat{u}-\zeta_{N, K}\right|_{\mathcal{U}_{s+2 \epsilon}^{1}} & =\left|\hat{u}-\zeta_{N, K}\right|_{\mathcal{U}_{0 ; \rho, s+2 \epsilon}^{1}} \\
& \leq \max \left\{M_{1}(\rho, 0, q), M_{2}(\rho, 0, q)\right\}|\hat{u}|_{\mathcal{U}_{q ; \rho, s+2 \epsilon}^{1}} .
\end{aligned}
$$

From Lemma 5.4 we have

$$
\left|\zeta_{N, K}-\zeta_{N, K}^{h}\right|_{\mathcal{U}_{s+2 \epsilon}^{1}} \leq C h^{\ell-1}\|\hat{u}\|_{\mathcal{U}_{s+2 \epsilon}^{\ell}} .
$$


Inserting (5.10) and (5.11) into (5.9) the lemma follows.

The following result follows from Corollary 4.6.

THEOREM 5.6. Consider the weights $\rho$ defined in (4.9). Under the assumptions of Lemma 5.5 we have for all $q>0$ that

$$
\left|\hat{u}-\hat{u}_{s}^{N, K, h}\right|_{\mathcal{U}_{s}^{1}} \leq C_{*}\left\{\max \left\{\frac{1}{\lambda_{1}^{N+1}}, \frac{1}{\lambda_{K+1}}\right\}^{q}|\hat{u}|_{\mathcal{U}_{q ; \rho, s+2 \epsilon}^{1}}+\hat{C} h^{\ell-1}\|\hat{u}\|_{\mathcal{U}_{s+2 \epsilon}^{\ell}}\right\},
$$

where $C_{*}=C_{*}(s, \epsilon)=1+e^{\frac{C_{\theta}^{2}}{\epsilon}} \prod_{j=K+1}^{\infty} \sigma_{j}(-s-\epsilon)$ and $\hat{C}$ is the constant of Lemma 5.4 .

From Corollary 4.9 we have the following a priori error estimate.

THEOREM 5.7. Consider the weights $\rho$ defined in (4.11). Under the assumptions of Lemma 5.5 we have for all $q>0$ that

$$
\left|\hat{u}-\hat{u}_{s}^{N, K, h}\right|_{\mathcal{U}_{s}^{1}} \leq C_{*}\left\{\max \left\{\frac{1}{1+(N+1) \lambda_{1}}, \frac{1}{1+\lambda_{K+1}}\right\}^{q}|\hat{u}|_{\mathcal{U}_{q ; \rho, s+2 \epsilon}^{1}}+\hat{C} h^{\ell-1}\|\hat{u}\|_{\mathcal{U}_{s+2 \epsilon}^{\ell}}\right\},
$$

where $C_{*}=C_{*}(s, \epsilon)=1+e^{\frac{C_{\theta}^{2}}{\epsilon}} \prod_{j=K+1}^{\infty} \sigma_{j}(-s-\epsilon)$ and $\hat{C}$ is the constant of Lemma 5.4 .

6. The resulting linear system. We now analyze the properties of the resulting linear system for the discrete spaces $\mathcal{X}_{s}^{N, K, h} \subset \widehat{\mathcal{U}}_{s}^{1}$ and $\mathcal{Y}_{s}^{N, K, h} \subset \widehat{\mathcal{U}}_{-(s+\epsilon)}^{1}$, defined in (5.1) and (5.2), respectively.

From (5.5), we see that for all functions $u \in \mathcal{X}_{s}^{N, K, h}$ and $v \in \mathcal{Y}_{s}^{N, K, h}$, i.e., $v(x, \omega)=$ $\tilde{v}(x, \omega) e^{\left(s+\frac{\epsilon}{2}\right)\left\|\Pi_{K} \omega\right\|_{-\theta}^{2}}$ with $\tilde{v} \in \mathcal{X}_{s}^{N, K, h}$ and $\Pi_{K}$ defined in (5.3), we have

(6.1) $a(u, v)=\int_{D} \hat{\kappa}_{K}(x) \int_{\mathcal{S}^{\prime}} e^{\sum_{j=1}^{K} a_{j}(x)\left\langle\omega, \eta_{j}\right\rangle} \nabla u(x, \omega) \nabla \tilde{v}(x, \omega) e^{\left(s+\frac{\epsilon}{2}\right)\left\|\Pi_{K} \omega\right\|_{-\theta}^{2}} d \mu(\omega) d x$,

where

$$
\hat{\kappa}_{K}(x):=e^{\frac{1}{2}\left(\left\|\phi_{x}\right\|_{H}^{2}-\sum_{j=1}^{K} a_{j}(x)^{2}\right)} .
$$

For every $u \in \mathcal{X}_{s}^{N, K, h}$ we introduce the function $\underline{u}: D \times \mathbb{R}^{K} \rightarrow \mathbb{R}$ such that

$$
\underline{u}\left(x,\left\langle\omega, \eta_{1}\right\rangle, \ldots,\left\langle\omega, \eta_{j}\right\rangle\right):=u(x, \omega) \text { for all } \omega \in \mathcal{S}^{\prime},
$$

and denote $\underline{\mathcal{X}}_{s}^{N, K, h}:=\left\{\underline{u}: \quad u \in \mathcal{X}_{s}^{N, K, h}\right\}$. We also introduce

$$
\kappa_{K}(x, y):=e^{\sum_{j=1}^{K} a_{j}(x) y_{j}}, \quad D_{K}:=\operatorname{diag}\left(\lambda_{1}^{-\theta}, \ldots, \lambda_{K}^{-\theta}\right)
$$

and define the bilinear form $\underline{a}$ by

$$
\underline{a}(\underline{u}, \underline{\tilde{v}}):=a(u, v) \text { for all } \underline{u}, \underline{\tilde{v}} \in \underline{\mathcal{X}}_{s}^{N, K, h} .
$$

Here $v(x, \omega)=\tilde{v}(x, \omega) e^{\left(s+\frac{\epsilon}{2}\right)\left\|\Pi_{K} \omega\right\|_{-\theta}^{2}}$. With this notation and using (6.1) we have

(6.4) $\underline{a}(\underline{u}, \underline{\tilde{v}})=\int_{D} \hat{\kappa}_{K}(x) \int_{\mathbb{R}^{K}} \kappa_{K}(x, y) \nabla_{x} \underline{u}(x, y) \nabla_{x} \underline{\tilde{v}}(x, y) e^{\left(s+\frac{\epsilon}{2}\right)\left|D_{K} y\right|^{2}} d \mu_{K}(y) d x$,

where $\mu_{K}$ denotes the standard Gaussian measure in $\mathbb{R}^{K}$. 
To simplify notation we set $\check{\sigma}=\sigma\left(s+\frac{\epsilon}{2}\right)$, i.e., let $\check{\sigma}_{j}=\sigma_{j}\left(s+\frac{\epsilon}{2}\right), j=1,2, \ldots$. Let $\left\{\psi_{\ell}\right\}_{\ell=1}^{L}$ be the standard hat basis functions for $X_{0}^{h}(D)$; then the collection

$$
\left\{\underline{\Psi}_{\ell, \check{\sigma}^{2}, \boldsymbol{\alpha}}: \quad \underline{\Psi}_{\ell, \check{\sigma}^{2}, \boldsymbol{\alpha}}(x, y)=\psi_{\ell}(x) \underline{H}_{\check{\sigma}^{2}, \boldsymbol{\alpha}}(y), \quad \ell=1, \ldots, L ; \quad \boldsymbol{\alpha} \in \mathcal{J}^{N, K}\right\}
$$

is a basis of $\underline{\mathcal{X}}_{s}^{N, K, h}$.

Denote by $\left\{A_{(\ell, \boldsymbol{\alpha}),(m, \boldsymbol{\beta})}\right\}$ the matrix associated with the bilinear form $\underline{a}$ defined in (6.3). From (6.4) we have

$$
\begin{aligned}
& A_{(\ell, \boldsymbol{\alpha}),(m, \boldsymbol{\beta})} \\
& =\int_{D} \hat{\kappa}_{K}(x) \int_{\mathbb{R}^{K}} \kappa_{K}(x, y) \underline{\Psi}_{\ell, \check{\sigma}^{2}, \boldsymbol{\alpha}}(x, y) \underline{\Psi}_{m, \check{\sigma}^{2}, \boldsymbol{\beta}}(x, y) e^{\left(s+\frac{\epsilon}{2}\right)\left|D_{K} y\right|^{2}} d \mu_{K}(y) d x \\
& =\int_{D} \hat{\kappa}_{K}(x) \kappa_{K, \boldsymbol{\alpha}, \boldsymbol{\beta}}^{\star}(x) \nabla \psi_{\ell}(x) \nabla \psi_{k}(x) d x,
\end{aligned}
$$

where we have defined

$$
\kappa_{K, \boldsymbol{\alpha}, \boldsymbol{\beta}}^{\star}(x):=\int_{\mathbb{R}^{K}} \kappa_{K}(x, y) \underline{H}_{\check{\sigma}^{2}, \boldsymbol{\alpha}}(y) \underline{H}_{\check{\sigma}^{2}, \boldsymbol{\beta}}(y) e^{\left(s+\frac{\epsilon}{2}\right)\left|D_{K} y\right|^{2}} d \mu_{K}(y) .
$$

Now we compute the integral in (6.7). From the definition of the $\check{\sigma}$-Fourier-Hermite polynomials we see that

$$
\begin{aligned}
\kappa_{K, \boldsymbol{\alpha}, \boldsymbol{\beta}}^{\star}(x) & =\prod_{j=1}^{K} \int_{\mathbb{R}} h_{\check{\sigma}_{j}^{2}, \alpha_{j}}\left(y_{j}\right) h_{\check{\sigma}_{j}^{2}, \beta_{j}}\left(y_{j}\right) e^{a_{j}(x) y_{j}} e^{\left(s+\frac{\epsilon}{2}\right) \lambda_{j}^{-2 \theta} y_{j}^{2}} d \mu_{1} \\
(6.8) \quad & =\prod_{j=1}^{K} \check{\sigma}_{j} \int_{\mathbb{R}} h_{\check{\sigma}_{j}^{2}, \alpha_{j}}\left(y_{j}\right) h_{\check{\sigma}_{j}^{2}, \beta_{j}}\left(y_{j}\right) e^{a_{j}(x) y_{j}} \frac{e^{-\frac{1}{2 \check{\sigma}_{j}^{2}} y_{j}^{2}}}{\sqrt{2 \pi} \check{\sigma}_{j}} d y_{j}=\prod_{j=1}^{K} \check{\sigma}_{j} \kappa^{\star}\left(x ; \alpha_{j}, \beta_{j}\right),
\end{aligned}
$$

where

$$
\kappa^{\star}\left(x ; \alpha_{j}, \beta_{j}\right):=\int_{\mathbb{R}} h_{\check{\sigma}_{j}^{2}, \alpha_{j}}\left(y_{j}\right) h_{\check{\sigma}_{j}^{2}, \beta_{j}}\left(y_{j}\right) e^{a_{j}(x) y_{j}} \frac{e^{-\frac{1}{2 \check{\sigma}_{j}^{2}} y_{j}^{2}}}{\sqrt{2 \pi} \check{\sigma}_{j}} d y_{j} .
$$

From the generating function identity (4.2) one can easily deduce

$$
h_{\check{\sigma}_{j}^{2}, \alpha_{j}}(t) h_{\check{\sigma}_{j}^{2}, \beta_{j}}(t)=\sum_{m=0}^{\min \left\{\alpha_{j}, \beta_{j}\right\}} m !\left(\begin{array}{c}
\alpha_{j} \\
m
\end{array}\right)\left(\begin{array}{c}
\beta_{j} \\
m
\end{array}\right) \check{\sigma}_{j}^{2 m} h_{\check{\sigma}_{j}^{2}, \alpha_{j}+\beta_{j}-2 m}(t)
$$

and

$$
e^{a_{j}(x) t}=e^{\frac{1}{2} \check{\sigma}_{j}^{2} a_{j}(x)^{2}} \sum_{m=0}^{\infty} \frac{1}{m !} a_{j}(x)^{m} h_{\check{\sigma}_{j}^{2}, m}(t)
$$

Then, we have

$(6.9) \kappa^{\star}\left(x ; \alpha_{j}, \beta_{j}\right)=e^{\frac{1}{2} \check{\sigma}_{j}^{2} a_{j}(x)^{2}} \sum_{m=0}^{\min \left\{\alpha_{j}, \beta_{j}\right\}} m !\left(\begin{array}{c}\alpha_{j} \\ m\end{array}\right)\left(\begin{array}{c}\beta_{j} \\ m\end{array}\right) a_{j}(x)^{\alpha_{j}+\beta_{j}-2 m} \check{\sigma}_{j}^{2\left(\alpha_{j}+\beta_{j}-m\right)}$. 
Summarizing, the $A_{(\ell, \boldsymbol{\alpha}),(m, \boldsymbol{\beta})}$ defined in (6.5) can be easily computed using (6.6), (6.2), and (6.7). This computation is reduced to the finite product in (6.8) where each factor is given by (6.9). For the proper computation of $\left\|\phi_{x}\right\|_{H}$, see section 7.2.

Denote by $\left\{g_{\ell, \boldsymbol{\alpha}}\right\}$ the load vector. Each entry is given by

$$
g_{\ell, \boldsymbol{\alpha}}=\int_{D \times \mathcal{S}^{\prime}} f(x, \omega) \psi_{\ell}(x) \prod_{j=1}^{d(\boldsymbol{\alpha})} h_{\check{\sigma}_{j}^{2}, \alpha_{j}}\left(\left\langle\omega, \eta_{j}\right\rangle\right) e^{\left(s+\frac{\epsilon}{2}\right)\left\|\Pi_{K} \omega\right\|_{-\theta}^{2}} d x d \mu(\omega) .
$$

The integral with respect the $\omega$ variable is exactly the computation of the $\boldsymbol{\alpha}$ th coefficient of the expansion of $f(x, \cdot)$ in terms of $\sigma\left(s+\frac{\epsilon}{2}\right)$-Fourier-Hermite polynomials. In particular if $f$ does not depend on $\omega$, then we have $g_{\ell, \boldsymbol{\alpha}}=0$ when $\boldsymbol{\alpha} \neq \mathbf{0}$.

Remark 6.1. It is easy to see that the matrix $\left\{A_{(\ell, \boldsymbol{\alpha}),(m, \boldsymbol{\beta})}\right\}$ associated with the bilinear form $\underline{a}$ is symmetric and positive definite. It is a square block matrix of dimension $\left(\begin{array}{c}K+{ }_{K} N \\ K\end{array}\right)$, where each block $(\boldsymbol{\alpha}, \boldsymbol{\beta})$ is the usual finite element matrix of the discretization of a second order equation with the coefficient given by $\hat{\kappa}(x) \kappa_{K}^{\star}(x ; \boldsymbol{\alpha}, \boldsymbol{\beta})$, where $b_{K}$ is defined in (6.2) and $\kappa_{K}^{\star}$ is defined in (6.7) and computed using (6.8) and (6.9). This corresponds to a discretization of a coupled system of second order equations. The resulting system is a very large sparse linear system of total dimension being $\left(\begin{array}{c}K+N \\ K\end{array}\right)$ times the dimension of a deterministic finite element problem. In the numerical experiment of section 8 we solve this system using a preconditioned conjugate gradient method with a block diagonal preconditioner. More efficient ways of solving this huge linear system will be the object of study in a future work.

Remark 6.2. Recall that we have set $\check{\sigma}_{j}=\sigma_{j}\left(s+\frac{\epsilon}{2}\right)$. The coefficients obtained from solving the resulting linear system are the coefficients of the numerical solution in terms of $\sigma\left(s+\frac{\epsilon}{2}\right)$-Fourier-Hermite polynomial basis. We can represent this solution in terms of the $\sigma(s)$-Fourier-Hermite polynomial basis using the following formula easily deduced from the generating function identity (4.2):

$$
\widehat{H}_{\check{\sigma}^{2}, \boldsymbol{\alpha}}(\omega)=\sum_{\gamma \leq \boldsymbol{\alpha} / 2} \frac{\boldsymbol{\alpha} !}{\gamma !(\boldsymbol{\alpha}-2 \gamma) !}\left(\frac{\sigma^{2}-\check{\sigma}^{2}}{2}\right)^{\gamma} \widehat{H}_{\sigma^{2}, \boldsymbol{\alpha}-2 \gamma}(\omega),
$$

where $\widehat{H}_{\sigma^{2}, \boldsymbol{\alpha}}(\omega):=\prod_{j=1}^{d(\boldsymbol{\alpha})} h_{\sigma_{j}^{2}, \alpha_{j}}\left(\left\langle\omega, \eta_{j}\right\rangle\right) \omega \in \mathcal{S}^{\prime}$. Note that this formula can also be used to deduce the expansion of the right-hand-side term $f$ in $\sigma\left(s+\frac{\epsilon}{2}\right)$-FourierHermite polynomials when $f$ is given in terms of another Fourier-Hermite polynomial basis.

7. On the choice of $\boldsymbol{H}, \boldsymbol{A}$, and $\phi_{x}$. Several choices for the Hilbert space $H$ and the operator $A$ are possible. We mention three possible choices of $\left(H, A, \phi_{x}\right)$. We will first review some known results.

\subsection{Known results.}

7.1.1. The Schwartz space and the operator $-\frac{d^{2}}{d x^{2}}+x^{2}+1$. Consider the densely defined differential operator

$$
A_{1}=-\frac{d^{2}}{d x^{2}}+x^{2}+1
$$

We have an $L^{2}(\mathbb{R})$ orthonormal system of eigenfunctions of $A_{1}$, which are the Hermite functions

$$
e_{n}(x):=\frac{1}{\sqrt{\sqrt{\pi}(n-1) !}} e^{-\frac{1}{2} x^{2}} h_{n-1}(\sqrt{2} x), \quad n=1,2, \ldots
$$


where $h_{n}$ is the $n$th degree Hermite polynomial. We have $A_{1} e_{n}=(2 n) e_{n}, n=1,2, \ldots$.

The family of tensors products

$$
e_{\boldsymbol{n}}:=e_{\left(n_{1}, \cdots, n_{d}\right)}:=e_{n_{1}} \otimes \cdots \otimes e_{n_{d}}, \quad \boldsymbol{n}=\left(n_{1}, \ldots, n_{d}\right) \in \mathbb{N}^{d},
$$

forms an orthonormal basis for $L^{2}\left(\mathbb{R}^{d}\right)$. Let $\boldsymbol{n}^{(j)}=\left(n_{1}^{(j)}, \ldots, n_{d}^{(j)}\right)$ be the $j$ th multiindex in some fixed ordering of all $d$-dimensional multi-indices $\boldsymbol{n}=\left(n_{1}, \ldots, n_{d}\right) \in \mathbb{N}^{d}$. We assume this ordering has the property that

$$
i<j \Longrightarrow \prod_{k=1}^{d}\left(2 n_{k}^{(i)}\right) \leq \prod_{k=1}^{d}\left(2 n_{k}^{(j)}\right) .
$$

Now define

$$
\eta_{j}:=e_{\boldsymbol{n}^{(j)}}=e_{n_{1}^{(j)}} \otimes \cdots \otimes e_{n_{d}^{(j)}}, j=1,2, \ldots
$$

We have $A_{1}^{\otimes d} \eta_{j}=\lambda_{j} \eta_{j}$, where

$$
\lambda_{j}:=\prod_{k=1}^{d}\left(2 n_{k}^{(j)}\right), \quad j=1,2, \ldots
$$

Note that $\boldsymbol{n}^{(1)}=(1, \ldots, 1) \in \mathbb{R}^{d}, \lambda_{1}=2^{d}$, and $1<\lambda_{1} \leq \lambda_{2} \leq \ldots$.

For the next result, see Holden et al. [21, Lemma 2.3.3].

Lemma 7.1 (Zhang). With $d(\boldsymbol{\alpha})$ defined in (4.1), $\sum_{\boldsymbol{\alpha} \in \mathcal{J}} \prod_{k=1}^{d(\boldsymbol{\alpha})}(2 k)^{-q \alpha_{k}}<\infty$ if and only if $q>1$.

Corollary 7.2. For $\left\{\lambda_{j}\right\}_{j=1}^{\infty}$ defined in (7.5) we have that $\sum_{j=1}^{\infty} \lambda_{j}^{-q}<\infty$ for all $q>1$.

Proof. For $\boldsymbol{\alpha} \in \mathcal{J}$ define $N_{Z}(\boldsymbol{\alpha}):=\#\left\{j: \alpha_{j} \neq 0\right\}$. Observe that

$$
\sum_{j=1}^{\infty} \lambda_{j}^{-q}=\sum_{\boldsymbol{n} \in \mathbb{N}^{d}} \prod_{k=1}^{d}\left(2 n_{k}\right)^{-q}=\sum_{N_{Z}(\boldsymbol{\alpha}) \leq d} \prod_{k=1}^{d(\boldsymbol{\alpha})}(2 k)^{-q \alpha_{k}}<\sum_{\boldsymbol{\alpha} \in \mathcal{J}} \prod_{k=1}^{d(\boldsymbol{\alpha})}(2 k)^{-q \alpha_{k}}<\infty,
$$

where $d(\boldsymbol{\alpha})$ is defined in (4.1) and $d$ is the dimension of $\mathbb{R}^{d}$.

7.1.2. The covariance integral operator on $L^{2}(D)$ and Mercer's theorem. Consider the covariance operator $Q: L^{2}(D) \rightarrow L^{2}(D)$ associated with $W(x, \omega)$, i.e., the integral operator with the symmetric positive kernel function $C(x, \hat{x})=$ $E_{\mu} W(x, \cdot) W(\hat{x}, \cdot)$. Let us assume that this kernel is square integrable and symmetric. Then the operator $Q$ is a compact operator in $L^{2}(D)$ and we let $\left\{\mu_{j}\right\}_{j=1}^{\infty}$ and $\left\{\zeta_{j}\right\}_{j=1}^{\infty}$ denote its eigenvalues and eigenfunctions. We have $\sum_{j} \mu_{j}^{2}<\infty$.

We recall that from Mercer's theorem (see Riesz and Sz.-Nagy [31]) we can write

$$
C(x, \hat{x})=\sum_{j=1}^{\infty} \mu_{j} \zeta_{j}(x) \zeta_{j}(\hat{x})
$$

For results on the decay of the eigenvalues, see Frauenfelder, Schwab, and Todor [13, Propositions 2.3, 2.5, and 2.6].

7.2. Three modeling choices. With the notation and review of section 7.1 we now mention three modeling choices. 
7.2.1. First choice. The first modeling choice we propose is as follows:

(1) The Hilbert space $H=L^{2}\left(\mathbb{R}^{d}\right)$.

(2) The operator $A=A_{1}^{\otimes d}$ with the sequence $\left\{\lambda_{j}\right\}_{j=1}^{\infty}$ in (7.5) and the eigenfunctions $\left\{\eta_{j}\right\}_{j=1}^{\infty}$ in (7.4).

(3) For all $x \in D$ we define $\phi_{x}(\hat{x}):=\phi(\hat{x}-x), \hat{x} \in \mathbb{R}^{d}$, where $\phi \in L^{2}\left(\mathbb{R}^{d}\right)$.

From Corollary 7.2 we can take any $\theta>\frac{1}{2}$ (independent of the dimension $d$ ). We have that $\left\|\phi_{x}\right\|_{H}=\|\phi(\cdot-x)\|_{L^{2}\left(\mathbb{R}^{d}\right)}=\|\phi\|_{L^{2}\left(\mathbb{R}^{d}\right)}$. In order to verify the assumption of Theorem 3.1 it is enough to take $\phi \in \mathcal{S}_{\theta}$. The functions $a_{j}$ defined in (5.6) are $a_{j}(x)=\left(\phi(\cdot-x), \eta_{j}\right)$ and, in general, can be computed using numerical integration. Typically, we need only compute $a_{j}(x)$ for some chosen quadrature points on each triangle of a triangulation of $D$. In explicit applications the test function or window $\phi$ can be chosen such that the diameter of the support of $\phi$ is the maximal distance within which $W_{\phi}\left(x_{1}, \cdot\right)$ and $W_{\phi}\left(x_{2}, \cdot\right)$ are correlated; see Holden et al. [21]. We also recall that the map $x \mapsto \phi_{x}$ may be chosen to match covariance function; see Roman and Sarkis [32].

It is easy to see that this choice implies that $\mathcal{S}=\mathcal{S}\left(\mathbb{R}^{d}\right)$ is the Schwartz space of rapidly decreasing functions and then $\mathcal{S}^{\prime}=\mathcal{S}^{\prime}\left(\mathbb{R}^{d}\right)$ is the space of tempered distributions. The triplet $\left(\mathcal{S}^{\prime}\left(\mathbb{R}^{d}\right), \mathcal{B}\left(\mathcal{S}^{\prime}\left(\mathbb{R}^{d}\right)\right), \mu\right)$ is called 1-dimensional white noise probability space. The smoothed white noise of Definition 2.3 is called the 1-dimensional d-parameter smoothed white noise. In section 8 we present numerical experiments using this setup for the case $d=1$.

7.2.2. Second choice. The second modeling choice we propose is as follows:

(1) The Hilbert space $H=L^{2}\left(\mathbb{R}^{d}\right)$.

(2) The operator $A=A_{1}^{\otimes d}$ with the sequence $\left\{\lambda_{j}\right\}_{j=1}^{\infty}$ in (7.5) and the eigenfunctions $\left\{\eta_{j}\right\}_{j=1}^{\infty}$ in $(7.4)$.

(3) For all $x \in D$ and $\hat{x} \in \mathbb{R}^{d}, \phi_{x}(\hat{x})=\sum_{j=1}^{\infty} a_{j}(x) \eta_{j}(\hat{x})$ and $a_{j}(x):=\sqrt{\mu_{j}} \zeta_{j}(x)$, where $\left\{\mu_{j}\right\}_{j=1}^{\infty}$ and $\left\{\zeta_{j}\right\}_{j=1}^{\infty}$ are the eigenvalues and eigenfunctions of the operator $Q$ introduced in section 7.1.2.

From (7.6) we see that $C(x, \hat{x})=\sum_{j=1}^{\infty} a_{j}(x) a_{j}(\hat{x})=\left(\phi_{x}, \phi_{\hat{x}}\right)_{H}, x, \hat{x} \in D$. Note also that $\left\|\phi_{x}\right\|_{H}^{2}=\left\|\phi_{x}\right\|_{L^{2}\left(\mathbb{R}^{d}\right)}^{2}=C(x, x)$ and

$$
\left\|\phi_{x}\right\|_{\theta}^{2}=\sum_{j=1}^{\infty} \lambda_{j}^{2 \theta} \mu_{j} \zeta_{j}(x)^{2} \text { for all } x \in D .
$$

The assumption $\phi_{x} \in \mathcal{S}_{\theta}$ for all $x \in D$ must be checked in each case, and the convergence of the series depends on the decay of the eigenvalues and on the $L^{\infty}(D)$-norm of the eigenfunctions, which in turn depend on the regularity of the function $C(x, \hat{x})$; see Frauenfelder, Schwab, and Todor [13, Propositions 2.3, 2.5, and 2.6]. For the numerical computation of the eigenfunctions and eigenvalues of $Q$, see Todor [36] and also Frauenfelder, Schwab, and Todor [13]. We also note that $\phi_{x}$ defined in (3) above can be used with any choice of Hilbert space $H$ and operator $A$. A possible choice is the one described next and which can be viewed as a generalization of the KL expansion.

7.2.3. Third choice. The third modeling choice we propose is as follows:

(1) The Hilbert space $H=L^{2}(D)$.

(2) $A=Q^{-1}$ with $\lambda_{j}:=\frac{1}{\mu_{j}}, j=1,2, \ldots$, and $\eta_{j}:=\zeta_{j}, j=1,2, \ldots$, where the $\mu_{j}$ and $\zeta_{j}$ are the eigenvalues and eigenfunctions of the integral operator $Q$.

(3) For all $x, \hat{x} \in D, \phi_{x}(\hat{x})=\sum_{j=1}^{\infty} a_{j}(x) \eta_{j}(\hat{x})$, where $a_{j}(x):=\sqrt{\mu_{j}} \zeta_{j}(x)$. 
According to section 7.1.2 we can take any $\theta \geq 1$. In this case the expansion of $W(x, \omega)$ in terms of $\sigma(s)$-Fourier-Hermite polynomials coincides with its KL expansion for the case $s=0$. We mention that in order to make calculations, such as writing the expansion of the right-hand side $f(x, \omega)$ in terms of $\sigma(s)$-Fourier-Hermite polynomials, we need to know the eigenfunctions of $Q$. We have $\left\|\phi_{x}\right\|_{H}^{2}=\left\|\phi_{x}\right\|_{L^{2}(D)}^{2}=C(x, x)$ and the assumption $\phi_{x} \in \mathcal{S}_{\theta}$ for all $x \in D$ must be checked for each particular problem. Observe also that $\left\|\phi_{x}\right\|_{\theta}^{2}=\sum_{j=1}^{\infty} \frac{1}{\mu_{j}^{2 \theta-1}} \zeta_{j}(x)^{2}$ for all $x \in D$.

8. Numerical experiments. In this section we present numerical experiments with $D=[0,1], H=L^{2}(\mathbb{R})$, and $A=A_{1}$ defined in (7.1) and we take $\theta=1$. In this case the eigenfunctions of $A$ correspond to the Hermite functions

$$
\eta_{j}(x)=\frac{1}{\sqrt{\sqrt{\pi}(j-1) !}} e^{-\frac{1}{2} x^{2}} h_{j-1}(\sqrt{2} x), \quad j=1,2, \ldots,
$$

where $h_{j}$ is the $j$ th degree Hermite polynomial. Also, since $\lambda_{1}^{2 \theta}=2$ for $\theta=1$, Lemma 2.2 becomes

$$
\int_{\mathcal{S}^{\prime}} e^{s\|\omega\|_{-\theta}^{2}} d \mu(\omega)=\left\{\begin{array}{lr}
\left(\frac{\sqrt{2}}{\pi \sqrt{-s}} \sinh \left(\frac{\pi \sqrt{-s}}{\sqrt{2}}\right)\right)^{-\frac{1}{2}}, & s<0, \\
1, & s=0, \\
\left(\frac{\sqrt{2}}{\pi \sqrt{s}} \sin \left(\frac{\pi \sqrt{s}}{\sqrt{2}}\right)\right)^{-\frac{1}{2}}, & 0<s<2, \\
+\infty, & s \geq 2 .
\end{array}\right.
$$

Therefore, we can construct the general $\sigma(s)$-Fourier-Hermite polynomials for $s<2$; see Lemma 4.2. We consider the modeling choice described in section 7.2.1, i.e., $\phi_{x}(\cdot)=\phi(\cdot-x)$. To avoid numerical integration errors in the computation of the functions $a_{j}(x)$ in (5.6), we choose the function $\phi$ as

$$
\phi(x)=e^{-\frac{1}{2} x^{2}}
$$

since explicit calculations can be performed, and therefore, the norm of the discrete errors can be calculated.

In order to compute the discretization errors, let $\hat{u}$ and $f$ be given by

$$
\hat{u}(x, \omega)=\frac{x(1-x)}{2} e^{-\left\langle\omega, \phi_{x}\right\rangle} \text { and } f(x, \omega)=1+\sum_{j=0}^{\infty}\left(\frac{x(1-x)}{2} a_{j}^{\prime}(x)\right)^{\prime}\left\langle\omega, \eta_{j}\right\rangle \text {. }
$$

It is easy to see that $\hat{u}$ in (8.2) is the exact solution of the problem (3.1) or (3.9) with the right-hand side $f$ given also in (8.2). By using the generating function identity (4.2) and direct calculations, the following results hold.

Lemma 8.1. For $\phi, \hat{u}$, and $f$ defined in (8.1) and (8.2), we have the following:

(1) $\|\phi\|_{L^{2}(\mathbb{R})}^{2}=\sqrt{\pi}$ and $\left\|\phi^{\prime}\right\|_{L^{2}(\mathbb{R})}^{2}=\frac{\sqrt{\pi}}{2}$.

(2) $a_{j}(x):=\left(\phi_{x}, \eta_{j}\right)=\sqrt{\frac{\sqrt{\pi}}{2^{j-1}(j-1) !}} x^{j-1} e^{-\frac{1}{4} x^{2}}$.

(3) The process $f$ belongs to $\mathcal{U}_{s}^{-1}$ for all $s<2$.

(4) $\hat{u}(x, \omega)=\sum_{\boldsymbol{\alpha} \in \mathcal{J}} \hat{u}_{\boldsymbol{\alpha}}(x) H_{\boldsymbol{\alpha}}(\omega) \in \widehat{\mathcal{U}}_{s}^{1}$ for all $s<2$, where

$$
\hat{u}_{\boldsymbol{\alpha}}(x)=e^{\frac{1}{2}\|\phi\|_{L^{2}(\mathbb{R})}^{2}} \frac{(-1)^{|\boldsymbol{\alpha}|} x(1-x) a_{\boldsymbol{\alpha}}(x)}{\boldsymbol{\alpha} !}
$$


with

$$
a_{\boldsymbol{\alpha}}(x)=\prod_{j=1}^{d(\boldsymbol{\alpha})} a_{j}(x)^{\alpha_{j}}=\left(\prod_{j=1}^{d(\boldsymbol{\alpha})} \sqrt{c_{j}}\right) x^{\sum_{j=1}^{d(\boldsymbol{\alpha})}(j-1) \alpha_{j}} e^{-\frac{1}{4}|\boldsymbol{\alpha}| x^{2}},
$$

where $c_{j}=\frac{\sqrt{\pi}}{2^{j-1}(j-1) !}$ for $j=1, \ldots$

(5) $\hat{u}(x, \omega)=\sum_{\boldsymbol{\alpha} \in \mathcal{J}^{\hat{u}_{\boldsymbol{\alpha}, s}}}(x) H_{\sigma(s)^{2}, \boldsymbol{\alpha}}$, where

$$
\hat{u}_{\boldsymbol{\alpha}, s}(x)=e^{\frac{1}{2} \sum_{j=1}^{\infty} \sigma_{j}(s)^{2} a_{j}(x)^{2}} \frac{(-1)^{|\boldsymbol{\alpha}|} x(1-x) a_{\boldsymbol{\alpha}}(x)}{\boldsymbol{\alpha} !} .
$$

(6) $|\hat{u}|_{\mathcal{U}_{0}^{1}}^{2}=\left(\frac{1}{12}+\frac{\left\|\phi^{\prime}\right\|_{L^{2}(\mathbb{R})}^{2}}{120}\right) e^{2\|\phi\|_{L^{2}(\mathbb{R})}^{2}}$.

(7) $C(x, \hat{x})=E_{\mu}\left\langle\cdot, \phi_{x}\right\rangle\left\langle\cdot, \phi_{\hat{x}}\right\rangle=\sqrt{\pi} e^{-\frac{1}{4}(x-\hat{x})^{2}}$.

Remark 8.2. It can be verified that the norm $\|\hat{u}\|_{\mathcal{U}_{q: \rho, s}^{1}}$ is finite if we use the weight of Example 4.5 with $\nu=0$.

Throughout this section, we solve the discrete problem (5.4) with $s=0$ to obtain

$$
\hat{u}^{N, K, h}(x, \omega)=\sum_{\boldsymbol{\alpha} \in \mathcal{J}^{N, K}} \hat{u}_{\boldsymbol{\alpha}}^{N, K, h}(x) H_{\boldsymbol{\alpha}}(\omega) .
$$

In Table 8.1 we show the seminorm error $\left|\hat{u}-\hat{u}^{N, K, h}\right|_{\mathcal{U}_{0}^{1}}$ for $\epsilon=1 / 2$ and $h=1 / 32$. Note that the condition $s+2 \epsilon<\lambda_{1}^{2 \theta} / 2$ of Lemma 5.5 is satisfied. We recall that this seminorm involves the computation of $\left|\hat{u}_{\boldsymbol{\alpha}}-\hat{u}_{\boldsymbol{\alpha}}^{N, K, h}\right|_{H^{1}(D)}^{2}$ for all $\boldsymbol{\alpha} \in \mathcal{J}^{N, K}$, and the computation of $\left|\hat{u}_{\boldsymbol{\alpha}}\right|_{H^{1}(D)}^{2}$ for all $\boldsymbol{\alpha} \in \mathcal{J} \backslash \mathcal{J}^{N, K}$. In this table we see clearly the decay of the errors with respect to $N$ and $K$.

We now analyze further the previous decay. Define the $a$-energy norm

$$
\left|\hat{u}-\hat{u}^{N, K, h}\right|_{a}:=a\left(\hat{u}-\hat{u}^{N, K, h}, \hat{u}-\hat{u}^{N, K, h}\right)^{\frac{1}{2}} .
$$

In Table 8.2, we present errors in the seminorm $|\cdot|_{\mathcal{U}_{0}^{1}}$ and in the $a$-energy norm $|\cdot|_{a}$. Here $h=1 / 16,1 / 32, \epsilon=1 / 2$, and $K=N=k$ for several values of $k$. The second row of Table 8.2 shows the number of Wiener-Chaos terms. The third row shows the $\mathcal{U}_{0}^{1}$-interpolation error and the rate of convergence (in parentheses) when we truncate the degree and the length of the polynomials. We can see a faster convergence rate when we increase $k$. This is somehow expected since $q$ can be taken as large as we wish in Theorem 5.6; see Remark 8.2. The fourth row shows the error between the discrete and the exact solutions in the $\mathcal{U}_{0}^{1}$ seminorm for $h=1 / 16$ and a line below for $h=1 / 32$; we also show the rate of convergence (in parentheses) for $h=1 / 16$. The decay now is not as fast as in the third row. This deterioration is due to the large value of $e^{C_{\theta} / \epsilon}$ that appears in the a priori error estimate in Lemma 5.5. The larger this constant is, the larger $k$ must be for observing a fast decay behavior. In order to minimize the effect of this constant, in the fifth row we measure the error in the $a$-energy norm and observe the fast decay. It was observed that the case $\epsilon=1 / 2$ shows a slightly better approximation, also in the $\mathcal{U}_{0}^{1}$ seminorm, than $\epsilon=0$ for small values of $k$. Finally, in Figure 8.1 we show the approximations of the mean value of the solution for different values of $\epsilon$. We observe that for larger $\epsilon$ values we obtain a better approximation of the mean value of the solution. Here we note, however, that when $\epsilon$ increases above $\epsilon=\frac{1}{2}$ the error in the $|\cdot|_{\mathcal{U}_{0}^{1}}$ norm deteriorates. Recall that the error in the $|\cdot|_{\mathcal{U}_{0}^{1}}$ norm includes the $H_{0}^{1}(0,1)$ error on the approximation of all coefficients $\hat{u}_{\boldsymbol{\alpha}}, \boldsymbol{\alpha} \in \mathcal{J}$. 
TABLE 8.1

Total error in the seminorm $|\cdot|_{\mathcal{U}_{0}^{1}}$. Here $h=1 / 32, \epsilon=\frac{1}{2}$.

\begin{tabular}{|c|c|c|c|c|c|c|}
\hline$K \downarrow N \rightarrow$ & 1 & 2 & 3 & 4 & 5 & 6 \\
\hline 1 & 1.6153 & 1.4358 & 1.2512 & 1.1108 & 1.0293 & 0.9918 \\
2 & 1.5970 & 1.3575 & 1.0672 & 0.7948 & 0.5896 & 0.4670 \\
3 & 1.5942 & 1.3446 & 1.0340 & 0.7299 & 0.4814 & 0.3084 \\
4 & 1.5938 & 1.3429 & 1.0296 & 0.7214 & 0.4669 & 0.2849 \\
5 & 1.5938 & 1.3426 & 1.0291 & 0.7206 & 0.4659 & $*$ \\
\hline
\end{tabular}

TABLE 8.2

Errors for $K=N=k, h=1 / 16,1 / 32$, and $\epsilon=\frac{1}{2}$. For $h=1 / 32$ we have added in parentheses the reduction factor, when passing to the next value of $k$, corresponding to the projection and finite element error in the seminorm $|\cdot|_{\mathcal{U}_{0}^{1}}$ and the finite element error in the a-energy norm.

\begin{tabular}{|c|c|c|c|c|c|c|}
\hline$k$ & 0 & 1 & 2 & 3 & 4 & 5 \\
\hline$\left(\begin{array}{c}K+N \\
K\end{array}\right)$ & 1 & 2 & 6 & 20 & 70 & 252 \\
\hline$\left|\hat{u}-Q^{N, K} \hat{u}\right|_{\mathcal{U}_{0}^{1}}$ & 1.6284 & $\begin{array}{l}1.3761 \\
(1.18)\end{array}$ & $\begin{array}{c}0.9767 \\
(1.41)\end{array}$ & $\begin{array}{c}0.6162 \\
(1.59)\end{array}$ & $\begin{array}{c}0.3570 \\
(1.73)\end{array}$ & $\begin{array}{c}0.1920 \\
(1.86)\end{array}$ \\
\hline$\left|\hat{u}-\hat{u}^{N, K, h}\right|_{\mathcal{U}_{0}^{1}}$ & $\begin{array}{l}1.7292 \\
1.7291\end{array}$ & $\begin{array}{l}1.6157 \\
1.6153 \\
(1.07)\end{array}$ & $\begin{array}{l}1.3590 \\
1.3575 \\
(1.18)\end{array}$ & $\begin{array}{l}1.0375 \\
1.0340 \\
(1.31)\end{array}$ & $\begin{array}{c}0.7281 \\
0.7214 \\
(1.43)\end{array}$ & $\begin{array}{c}0.4626 \\
0.4659 \\
(1.55)\end{array}$ \\
\hline$\left|\hat{u}-\hat{u}^{N, K, h}\right|_{a}$ & $\begin{array}{l}0.4319 \\
0.4318\end{array}$ & $\begin{array}{c}0.3691 \\
0.3688 \\
(1.17)\end{array}$ & $\begin{array}{c}0.2598 \\
0.2589 \\
(1.42)\end{array}$ & $\begin{array}{c}0.1573 \\
0.1552 \\
(1.67)\end{array}$ & $\begin{array}{c}0.0836 \\
0.0790 \\
(1.96)\end{array}$ & $\begin{array}{c}0.0454 \\
0.0279 \\
(2.83)\end{array}$ \\
\hline
\end{tabular}

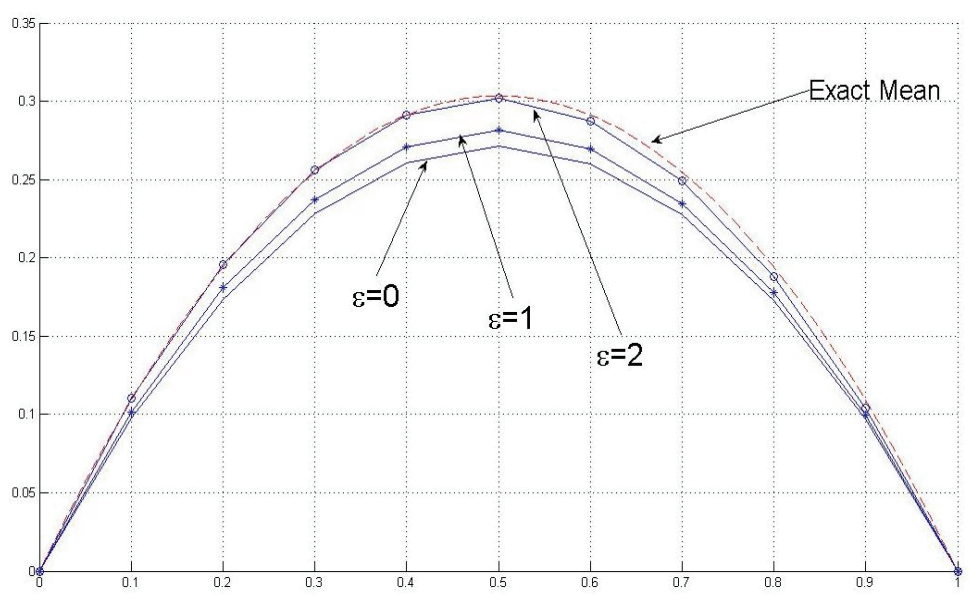

FIG. 8.1. Approximation of the coefficient $u_{(0,0,0, \ldots)}$ for $K=N=3, h=\frac{1}{10}$, and $\epsilon=0,1,2$.

9. Conclusions and final comments. We consider the white noise calculus constructed from a Hilbert space and an operator in order to describe the white noise probability space and to study the pressure equation with stochastic coefficients and forcing terms. By introducing appropriate spaces for the solution and test functions for the weak formulation of the problem, we prove the continuous inf-sup condition and establish existence and uniqueness of the solution of the problem. Finite dimensional discretizations are introduced for this problem, which are based on finite element methods, truncated Wiener-Chaos expansions, and exponential stochastic 
functions. By introducing appropriate discrete spaces and norms (Sobolev for the spatial component and Hida-Kuo-Kondratiev-Streit type for the stochastic components) we establish existence and uniqueness of the solution and provide a priori error estimates. Finally, we choose a particular model to numerically validate the proposed discretizations.

Acknowledgment. We would like to thank the referees for their useful comments, which helped to improve the presentation of this paper.

\section{REFERENCES}

[1] I. BabušKa And P. Chatzipantelidis, On solving elliptic stochastic partial differential equations, Comput. Methods Appl. Mech. Engrg., 191 (2002), pp. 4093-4122.

[2] I. BabušKa, F. Nobile, And R. Tempone, A stochastic collocation method for elliptic partial differential equations with random input data, SIAM J. Numer. Anal., 45 (2007), pp. 10051034 .

[3] I. BABušKa, R. TEMPOne, AND G. E. Zouraris, Galerkin finite element approximations of stochastic elliptic partial differential equations, SIAM J. Numer. Anal., 42 (2004), pp. 800825.

[4] F. E. Benth and J. Gjerde, Convergence rates for finite element approximations of stochastic partial differential equations, Stochastics Stochastics Rep., 63 (1998), pp. 313-326.

[5] F. E. Benth And T. G. Theting, Some regularity results for the stochastic pressure equation of Wick-type, Stochastic Anal. Appl., 20 (2002), pp. 1191-1223.

[6] Y. M. Berezanskĭ, Selfadjoint Operators in Spaces of Functions of Infinitely Many Variables, Transl. Math. Monogr. 63, American Mathematical Society, Providence, RI, 1986 (translated from the Russian by H. H. McFaden; translation edited by Ben Silver).

[7] V. I. Bogachev, Gaussian measures, Math. Surveys Monogr. 62, American Mathematical Society, Providence, RI, 1998.

[8] C. Canuto and T. Kozubek, A fictitious domain approach to the numerical solution of PDEs in stochastic domains, Numer. Math., 107 (2007), pp. 257-293.

[9] Y. CAO, On convergence rate of Wiener-Ito expansion for generalized random variables, Stochastics, 78 (2006), pp. 179-187.

[10] W. G. Cochran, H.-H. Kuo, And A. Sengupta, A new class of white noise generalized functions, Infin. Dimens. Anal. Quantum Probab. Relat. Top., 1 (1998), pp. 43-67.

[11] G. Da Prato, An Introduction to Infinite-Dimensional Analysis, revised and extended from the 2001 original by Da Prato, Universitext, Springer-Verlag, Berlin, 2006.

[12] G. Da Prato And J. ZabczyK, Stochastic Equations in Infinite Dimensions, Encyclopedia Math. Appl. 44, Cambridge University Press, Cambridge, 1992.

[13] P. Frauenfelder, C. Schwab, And R. A. Todor, Finite elements for elliptic problems with stochastic coefficients, Comput. Methods Appl. Mech. Engrg., 194 (2005), pp. 205-228.

[14] F. Furtado and F. Pereira, Crossover from nonlinearity controlled to heterogeneity controlled mixing in two-phase porous media flows, Comput. Geosci., 7 (2003), pp. 115-135.

[15] J. Galvis And M. Sarkis, Regularity Results for the Ordinary Product Stochastic Pressure Equation, in preparation.

[16] R. G. Ghanem, Ingredients for a general purpose stochastic finite elements implementation, Comput. Methods Appl. Mech. Engrg., 168 (1999), pp. 19-34.

[17] R. G. Ghanem and R. Kruger, Numerical solution of spectral stochastic finite element systems, Comput. Methods Appl. Mech. Engrg., 129 (1996), pp. 289-303.

[18] R. G. Ghanem and P. D. Spanos, Stochastic Finite Elements: A Spectral Approach, SpringerVerlag, New York, 1991.

[19] T. HidA, Brownian Motion, Applications of Mathematics 11, Springer-Verlag, New York, 1980 (translated from the Japanese by the author and T. P. Speed).

[20] T. Hida, H.-H. Kuo, J. Potthoff, and L. Streit, White Noise. An Infinite-Dimensional Calculus, Math. Appl. 253, Kluwer Academic Publishers, Dordrecht, 1993.

[21] H. Holden, B. Øksendal, J. Ubøe, and T. Zhang, Stochastic Partial Differential Equations: A Modeling, White Noise Functional Approach, Probab. Appl., Birkhäuser Boston, Boston, 1996.

[22] C. Jin, X.-C. CAI, AND C. LI, Parallel domain decomposition methods for stochastic elliptic equations, SIAM J. Sci. Comput., 29 (2007), pp. 2096-2114. 
[23] A. KeEse, A Review of Recent Developments in the Numerical Solution of Stochastic Partial Differential Equations (Stochastic Finite Elements), Technical report 2003-06, Institute of Scientific Computing, Technical University Braunschweig, Informatikbericht, Braunschweig, Germany, 2003.

[24] H.-H. Kuo, Gaussian Measures in Banach Spaces, Lecture Notes in Math. 463, Springer-Verlag, Berlin, 1975.

[25] H.-H. Kuo, White Noise Distribution Theory, Probab. Stochastics Ser., CRC Press, Boca Raton, FL, 1996.

[26] H. G. MatthiEs, Stochastic finite elements: Computational approaches to stochastic partial differential equations, ZAMM Z. Angew. Math. Mech., 88 (2008), pp. 849-873.

[27] H. G. Matthies And A. Keese, Galerkin methods for linear and nonlinear elliptic stochastic partial differential equations, Comput. Methods Appl. Mech. Engrg., 194 (2005), pp. 12951331.

[28] F. Nobile, R. Tempone, and C. G. Webster, An anisotropic sparse grid stochastic collocation method for partial differential equations with random input data, SIAM J. Numer. Anal., 46 (2008), pp. 2411-2442.

[29] F. Nobile, R. Tempone, And C. G. Webster, A sparse grid stochastic collocation method for partial differential equations with random input data, SIAM J. Numer. Anal., 46 (2008), pp. 2309-2345.

[30] N. OвAтA, White Noise Calculus and Fock Space, Lecture Notes in Math. 1577, SpringerVerlag, Berlin, 1994.

[31] F. Riesz And B. Sz.-NAgy, Functional Analysis, reprint of the 1955 original, Dover Books Adv. Math., Dover, New York, 1990 (translated from the second French edition by Leo F. Boron).

[32] L. J. Roman And M. Sarkis, Stochastic Galerkin method for elliptic SPDEs: A white noise approach, Discrete Contin. Dyn. Syst. Ser. B, 6 (2006), pp. 941-955.

[33] C. Schwab And R. A. Todor, Sparse finite elements for stochastic elliptic problems-higher order moments, Computing, 71 (2003), pp. 43-63.

[34] I. ShigeKawa, Stochastic Analysis, Iwanami Series in Modern Mathematics, Transl. Math. Monogr. 224, American Mathematical Society, Providence, RI, 2004 (translated from the 1998 Japanese original by the author).

[35] T. G. Theting, Solving Wick-stochastic boundary value problems using a finite element method, Stochastics Stochastics Rep., 70 (2000), pp. 241-270.

[36] R. A. Todor, Robust eigenvalue computation for smoothing operators, SIAM J. Numer. Anal., 44 (2006), pp. 865-878.

[37] D. XIU And G. E. Karniadakis, Modeling uncertainty in steady state diffusion problems via generalized polynomial chaos, Comput. Methods Appl. Mech. Engrg., 191 (2002), pp. 49274948.

[38] D. Xiu And G. E. KaRniadakis, The Wiener-Askey polynomial chaos for stochastic differential equations, SIAM J. Sci. Comput., 24 (2002), pp. 619-644. 